\title{
ON BEHAVIOR OF PAIRS OF TEICHMÜLLER GEODESIC RAYS
}

\begin{abstract}
MASANORI AMANO
Abstract. In this paper, we obtain the explicit limit value of the Teichmüller distance between two Teichmüller geodesic rays which are determined by Jenkins-Strebel differentials having a common end point in the augmented Teichmüller space. Furthermore, we also obtain a condition under which these two rays are asymptotic. This is similar to a result of Farb and Masur.
\end{abstract}

\section{INTRODUCTION}

Let $T(X)$ be the Teichmüller space of an analytically finite Riemann surface $X$. Each Teichmüller geodesic ray on $T(X)$ is determined by a holomorphic quadratic differential on an initial point. We are interested in the behavior of two geodesic rays near the boundary of the Teichmüller space.

An interesting question is, what happens when the Teichmüller distance between the given two Teichmüller geodesic rays are bounded, or diverge. This question is answered completely by Ivanov [Iva01, Lenzhen and Masur [LM10] and Masur Mas75, Mas80. The details are the following. First, Masur showed that if the measured foliations of the given rays are Jenkins-Strebel and topologically equivalent, then the rays are bounded ([Mas75]). Masur also showed that if the measured foliations are uniquely ergodic and topologically equivalent with the condition that they have no simple closed curves consist of saddle connections, then the rays are asymptotic (and so, bounded) ([Mas80]). Ivanov showed that if the measured foliations are absolutely continuous, then the rays are bounded, and if the measured foliations have non-zero intersection number, then the rays are divergent ([Iva01]). Finally, Lenzhen and Masur showed that if the measured foliations are not absolutely continuous, or the measured foliations are not topologically equivalent and have zero intersection number, then the rays are divergent ([LM10]).

On the other hand, Farb and Masur FM10 considered two Jenkins-Strebel rays in the moduli space given by modularly equivalent holomorphic quadratic differentials. They showed that under some conditions of initial points of two rays, the limit value of the distance between the rays in the moduli space is the distance between the end points of the rays in the boundary of the moduli space. If, in addition, the end points coincide, then they are asymptotic.

In this paper, we consider two Teichmüller geodesic rays, $r, r^{\prime}: \mathbb{R}_{\geq 0} \rightarrow T(X)$ on the Teichmüller space $T(X)$ starting at $r(0)=[Y, f], r^{\prime}(0)=\left[Y^{\prime}, \bar{f}^{\prime}\right]$ and having

Received by the editors April 18, 2013 and, in revised form, August 7, 2013 and September $11,2013$.

2010 Mathematics Subject Classification. Primary 32G15; Secondary 30F60.

Key words and phrases. Teichmüller space, Teichmüller distance, Teichmüller geodesic, augmented Teichmüller space, Gardiner-Masur compactification, horofunction. 
initial unit norm Jenkins-Strebel differentials $q, q^{\prime}$ on $Y, Y^{\prime}$, respectively. Let $r(\infty)$, $r^{\prime}(\infty)$ be the end points of $r, r^{\prime}$ in the augmented Teichmüller space, respectively. Let $H(q), H\left(q^{\prime}\right)$ be measured foliations corresponding to $q, q^{\prime}$, respectively, and we suppose that the measured foliations $f_{*}^{-1}(H(q)), f_{*}^{\prime-1}\left(H\left(q^{\prime}\right)\right)$ are topologically equivalent. We denote by $m_{1}, \cdots, m_{k}, m_{1}^{\prime}, \cdots, m_{k}^{\prime}$ the moduli of annuli determined by $f_{*}^{-1}(H(q)), f_{*}^{\prime-1}\left(H\left(q^{\prime}\right)\right)$, respectively. Under the above assumption, we determine the limit value of the Teichmüller distance between two points $r(t), r^{\prime}(t)$.

Theorem 1.1. If $r(\infty)=r^{\prime}(\infty)$, then

$$
\lim _{t \rightarrow \infty} d_{T(X)}\left(r(t), r^{\prime}(t)\right)=\frac{1}{2} \log \max _{j=1, \cdots, k}\left\{\frac{m_{j}^{\prime}}{m_{j}}, \frac{m_{j}}{m_{j}^{\prime}}\right\} .
$$

Furthermore, we obtain the following condition which is equivalent to the asymptoticity of two Jenkins-Strebel rays. This is similar to a theorem of Farb and Masur [FM10].

Corollary 1.2. For any two Jenkins-Strebel rays $r, r^{\prime}$, they are asymptotic if and only if $r, r^{\prime}$ are modularly equivalent and $r(\infty)=r^{\prime}(\infty)$.

In Theorem 1.1, the limit value depends on the modulus of each annulus which is determined by the initial holomorphic quadratic differentials on the initial points $r(0), r^{\prime}(0)$ of the given rays. Therefore, this value depends on the choice of the initial points $r(0), r^{\prime}(0)$. We consider the minimum of the limit value of the Teichmüller distance between two rays when we shift the initial points of the rays. We show that the minimum is represented by the detour metric $\delta$ between the end points of the rays in the Gardiner-Masur boundary of $T(X)$.

Proposition 1.3. Under the assumption of Theorem 1.1, the minimum of the limit value of the distance between the given rays $r(t), r^{\prime}(t)$ when we shift the initial points $r(0), r^{\prime}(0)$ is given by $\frac{1}{2} \delta\left(\xi, \xi^{\prime}\right)$, where $\xi, \xi^{\prime}$ are the end points of the rays $r$, $r^{\prime}$ in the Gardiner-Masur boundary of $T(X)$, respectively.

\section{BACKGROUND}

2.1. Teichmüller spaces. Let $X$ be a Riemann surface of type $(g, n)$ with $3 g-3+$ $n>0$. The Teichmüller space $T(X)$ of $X$ is the set of all equivalence classes $[Y, f]$ of pairs of a Riemann surface $Y$ and a quasiconformal mapping $f: X \rightarrow Y$. Two pairs $\left(Y_{1}, f_{1}\right)$ and $\left(Y_{2}, f_{2}\right)$ are equivalent if there is a conformal mapping $h: Y_{1} \rightarrow Y_{2}$ such that $h \circ f_{1}$ is homotopic to $f_{2}$. The Teichmüller space $T(X)$ has a complete distance, called the Teichmüller distance $d_{T(X)}$. For any $p_{1}=\left[Y_{1}, f_{1}\right], p_{2}=\left[Y_{2}, f_{2}\right] \in T(X)$, the distance is defined by

$$
d_{T(X)}\left(p_{1}, p_{2}\right)=\frac{1}{2} \inf _{h} \log K(h),
$$

where $h$ ranges over all quasiconformal mappings $h: Y_{1} \rightarrow Y_{2}$ such that $h \circ f_{1}$ is homotopic to $f_{2}$, and $K(h)$ means the maximal quasiconformal dilatation of $h$.

2.2. Holomorphic quadratic differentials. In this section, we refer to [Str84] for details.

A holomorphic quadratic differential $q$ on $X$ is a tensor which is represented locally by $q=q(z) d z^{2}$, where $q(z)$ is a holomorphic function of the local coordinate $z=x+i y$ on $X$. We allow holomorphic quadratic differentials to have simple poles at the punctures of $X$. For each holomorphic quadratic differential $q=$ 
$q(z) d z^{2},|q|$ is defined locally by the differential 2-form $|q(z)| d x d y$ on each coordinate $z$. The norm of $q$ is defined by $\|q\|=\iint_{X}|q(z)| d x d y$. We treat holomorphic quadratic differentials whose norms are finite. A holomorphic quadratic differential $q$ is called of unit norm if it satisfies $\|q\|=1$. A critical point of $q$ is a zero of $q$ or a puncture of $X$. Each non-zero holomorphic quadratic differential has finitely many critical points. In a neighborhood of any non-critical point of $q$, there exists a local coordinate $\zeta$ on $X$ such that $q=d \zeta^{2}$. Indeed, let $p_{0}$ be a non-critical point; then $q^{\frac{1}{2}}=q(z)^{\frac{1}{2}} d z$ has a single-valued holomorphic branch in some neighborhood $U$ around $p_{0}$. The new coordinate

$$
\zeta(p)=\int_{p_{0}}^{p} q^{\frac{1}{2}}
$$

is defined for any $p \in U$, and this coordinate satisfies $q=d \zeta^{2}$. We call the coordinate $\zeta$ a $q$-coordinate on $X$. For any two $q$-coordinates $\zeta_{1}, \zeta_{2}$ in a common neighborhood $U$, the equation $\zeta_{2}= \pm \zeta_{1}+c$ hold where $c \in \mathbb{C}$, because $q=d \zeta_{1}^{2}=d \zeta_{2}^{2}$. A smooth path $z=\gamma(t)$ on $X$ is called a horizontal trajectory of $q$ if it is a maximal path which satisfies $q(\gamma(t)) \dot{\gamma}(t)^{2}>0$. We notice that any horizontal trajectory of $q$ consists of non-critical points of $q$, and it is represented by a Euclidean horizontal segment in $q$-coordinates. All horizontal trajectories of $q$ are classified by the following three types. A horizontal trajectory of $q$ is critical if it joins critical points of $q$, closed if it is a closed path, recurrent otherwise. The recurrent trajectory is dense on a subsurface of $X$ whose boundary consists of critical trajectories of $q$. Let $\Gamma_{q}$ be the set of all critical points and critical trajectories of $q$. Any component of $X-\Gamma_{q}$ is an annulus which is swept out by closed trajectories of $q$ such that they are homotopic to each other, or a minimal domain which consists of infinitely many recurrent trajectories of $q$. If all components of $X-\Gamma_{q}$ are annuli, we call $q$ a Jenkins-Strebel differential.

2.3. Measured foliations. In this section, we refer to [FLP79 for details.

A foliation $F=\left\{\left(U_{j}, z_{j}\right)\right\}_{j}$ with singularities on $X$ is an atlas of $X$, and is given by the pair of an open covering $\left\{U_{j}\right\}_{j}$ of $X-\{$ finite distinguished points $\}$ and each local coordinate $z_{j}=x_{j}+i y_{j}$ on $U_{j}$ such that the equations $x_{k}=f_{j k}\left(z_{j}\right)$, $y_{k}= \pm y_{j}+c$ hold where $c \in \mathbb{C}$ if $U_{j} \cap U_{k} \neq \emptyset$. These distinguished points and punctures of $X$ are called singularities of $F$. They are $p$-pronged singularities for $p \geq 1$, but the case of $p=1,2$ is attained only at the punctures of $X$. A maximal horizontal segment with respect to $\left\{z_{j}\right\}_{j}$ is called a leaf of $F$. A transverse measure $\mu$ of $F$ is given by a measure on the set of all transversal arcs of leaves of $F$ such that if transversal $\operatorname{arcs} \alpha, \beta$ are moved to each other by a homotopy along leaves of the foliation $F$, then $\mu(\alpha)=\mu(\beta)$. The pair $(F, \mu)$ is called a measured foliation on $X$. Let $\mathcal{S}$ be the set of all homotopy classes of non-trivial and non-peripheral simple closed curves on $X$. For any measured foliation $(F, \mu)$ and any $\alpha \in \mathcal{S}$, we can define the intersection number

$$
i((F, \mu), \alpha)=\inf _{\alpha^{\prime} \in \alpha} \int_{\alpha^{\prime}} d \mu
$$

where $\alpha^{\prime}$ ranges over all simple closed curves in $\alpha$. Two measured foliations $\left(F_{1}, \mu_{1}\right)$ and $\left(F_{2}, \mu_{2}\right)$ are equivalent if the equation

$$
i\left(\left(F_{1}, \mu_{1}\right), \alpha\right)=i\left(\left(F_{2}, \mu_{2}\right), \alpha\right)
$$

holds for any $\alpha \in \mathcal{S}$. We denote by $\mathcal{M F}(X)$ the set of all equivalence classes of measured foliations on $X$. The set $\mathcal{M F}(X)$ has the weak topology which is induced 
by intersection number functions in $\mathbb{R}_{\geq 0}^{\mathcal{S}}$. We denote by $[F, \mu]$ the equivalence class of $(F, \mu)$. We consider the space of measured foliations $\mathcal{M F}(Y)$ on any other Riemann surface $Y$ of the same type as $X$. For any homeomorphism $f: X \rightarrow$ $Y$, there exists a homeomorphism $f_{*}: \mathcal{M F}(X) \rightarrow \mathcal{M F}(Y)$ which is defined by $f_{*}([F, \mu])=\left[f(F), \mu \circ f^{-1}\right] \in \mathcal{M F}(Y)$ for any $[F, \mu] \in \mathcal{M F}(X)$. After this, we denote by $\mu$ the equivalence class of a measured foliation $[F, \mu] \in \mathcal{M F}(X)$. We can see that $\mathbb{R}_{\geq 0} \times \mathcal{S} \subset \mathcal{M F}(X)$. For any $b \geq 0$ and any $\gamma \in \mathcal{S}$, the measured foliation $b \gamma$ consists of closed leaves which are homotopic to $\gamma$, and leaves tend to singular points. These closed leaves produce an annulus of height $b$, and other leaves are the boundary of it. This measured foliation satisfies $i(b \gamma, \alpha)=b i(\gamma, \alpha)$ for any $\alpha \in \mathcal{S}$. The right side of this equation is a geometric intersection number of simple closed curves. Thurston showed that $\overline{\mathbb{R}_{>0} \times \mathcal{S}}=\mathcal{M F}(X)$. (For instance, we refer to FLP79.) Then, we can see that intersection number functions are defined on $\mathcal{M F}(X) \times \mathcal{M} \mathcal{F}(X)$. (We refer to Ree81 for details.) We notice that for any homeomorphism $f: X \rightarrow Y$ and any $\mu, \nu \in \mathcal{M F}(X)$, the equation $i\left(f_{*}(\mu), f_{*}(\nu)\right)=$ $i(\mu, \nu)$ holds.

Remark. The set $\mathcal{M F}(X)$ contains the zero-measured foliation, denoted by 0 ; that is, it has the zero intersection number with all measured foliations. Of course, for any $\mu \in \mathcal{M F}(X)$, we regard the measured foliation $0 \mu$ as 0 .

2.4. Measured foliations and holomorphic quadratic differentials. For any non-zero holomorphic quadratic differential $q$ on $X$, we can define the measured foliation $H(q) \in \mathcal{M F}(X)$ consisting of all horizontal trajectories of $q$ as leaves and $|d y|$ as a transverse measure, where $z=x+i y$ is the $q$-coordinate. The singularities of $H(q)$ are the critical points of $q$.

Remark. There is a one-to-one correspondence between the set of all holomorphic quadratic differentials of finite norm on $X$ and $\mathcal{M F}(X)$; we refer to [HM79].

If $q$ has an annulus $A$ in $X-\Gamma_{q}$, then the restriction to $A$ of the measured foliation $H(q)$ can be written as $\left.H(q)\right|_{A}=b \gamma$, where $\gamma \in \mathcal{S}$ corresponds to closed trajectories which sweep out in $A$, and $b>0$ is the height of $A$ with respect to the metric $|d y|$. If $q$ has a minimal domain $M$ in $X-\Gamma_{q}$, then the restriction to $M$ of the measured foliation $H(q)$ can be written as $\left.H(q)\right|_{M}=\sum_{i=1}^{p} b_{i} \mu_{i}$, where $p>0$ is bounded by the number which is determined by the topology of $M, b_{i} \geq 0$ for any $i=1, \cdots, p$ and $\left\{\mu_{i}\right\}_{i=1}^{p}$ is the set of ergodic transverse measures which are projectively-distinct and pairwise having zero intersection number (that is, for any $i \neq i^{\prime}$ and any $k \geq 0, \mu_{i^{\prime}} \neq k \mu_{i}$ and $\left.i\left(\mu_{i}, \mu_{i^{\prime}}\right)=0\right)$. A transverse measure $\mu$ is called an ergodic transverse measure if it is non-zero and cannot be written as a sum of projectively-distinct and non-zero measured foliations. (For ergodicity, we refer to KH95 for more information.) Any non-zero holomorphic quadratic differential $q$ has finitely many critical points, so the measured foliation $H(q)$ has finitely many annuli or minimal domains. Therefore, the measured foliation $H(q)$ can be written as

$$
H(q)=\sum_{j=1}^{k} b_{j} G_{j},
$$

where $G_{j}$ is (a homotopy class of) a simple closed curve or an ergodic measure for any $j=1, \cdots, k$ such that these are projectively-distinct and pairwise having zero intersection number, and $b_{j}>0$ if $G_{j} \in \mathcal{S}, b_{j} \geq 0$ if $G_{j}$ is an ergodic measure. 
(About this notation, we refer the reader to [Iva92 for more details.) In particular, if $q$ is a Jenkins-Strebel differential, then we can write

$$
H(q)=\sum_{j=1}^{k} b_{j} \gamma_{j}
$$

where $b_{1}, \cdots, b_{k}$ are positive real numbers and $\gamma_{1}, \cdots, \gamma_{k}$ are distinct simple closed curves such that $i\left(\gamma_{j}, \gamma_{j^{\prime}}\right)=0$ for any $j \neq j^{\prime}$, and in this situation, we also say that $H(q)$ is Jenkins-Strebel. If $X-\Gamma_{q}$ has only one minimal domain and the measured foliation $H(q)$ is represented by $b \mu$, where $b>0$ and $\mu$ is an ergodic measure, and any other topologically equivalent measured foliation $\nu$ on $X$ is represented by $\nu=b^{\prime} \mu$, where $b^{\prime}>0$, then $q$ and $H(q)$ are called uniquely ergodic. We come back to the general case and see that

$$
i\left(\sum_{j=1}^{k} b_{j} G_{j}, \alpha\right)=\sum_{j=1}^{k} b_{j} i\left(G_{j}, \alpha\right)
$$

for any $\alpha \in \mathcal{S}$.

Remark. If $G_{j}=\mu_{j}$ is ergodic, we regard $G_{j}$ as $\left[G, \mu_{j}\right] \in \mathcal{M F}(X)$, where $G$ is an unmeasured foliation which is $H(q)$ without the transverse measure. $\left(\mu_{j}\right.$ is supported on a minimal domain.) Then $G_{j}$ is a measured foliation on the whole surface $X$.

For any $j=1, \cdots, k$, we set

$$
m_{j}=\frac{b_{j}}{i\left(G_{j}, V(q)\right)},
$$

where the measured foliation $V(q)$ is defined by $H(-q)$. If $G_{j}$ is a simple closed curve $\gamma_{j} \in \mathcal{S}$, then $a_{j}:=i\left(\gamma_{j}, V(q)\right)$ means the infimum of the horizontal lengths of the simple closed curves in $\gamma_{j}$ with respect to the metric $|d x|$, and $m_{j}=\frac{b_{j}}{a_{j}}$ means a modulus of the annulus which is generated by $\gamma_{j}$, that is, the ratio of the height and the circumference of the annulus.

Let $q, q^{\prime}$ be unit norm holomorphic quadratic differentials on $X$ and $H(q), H\left(q^{\prime}\right)$ being measured foliations in $\mathcal{M F}(X)$ constructed by $q, q^{\prime}$, respectively.

Definition 2.1. The pair of holomorphic quadratic differentials $q, q^{\prime}$ on $X$ or measured foliations $H(q), H\left(q^{\prime}\right) \in \mathcal{M F}(X)$ is called topologically equivalent if there is a homeomorphism $\alpha: X-\Gamma_{q} \rightarrow X-\Gamma_{q^{\prime}}$ which is homotopic to the identity such that the leaves of $H(q)$ are mapped to the leaves of $H\left(q^{\prime}\right)$. In this situation, we can write the measured foliations as $H(q)=\sum_{j=1}^{k} b_{j} G_{j}, H\left(q^{\prime}\right)=\sum_{j=1}^{k} b_{j}^{\prime} G_{j}$, where $G_{j}$ is a simple closed curve or an ergodic measure for any $j=1, \cdots, k$ such that these are projectively-distinct and pairwise having zero intersection number, and $b_{j}, b_{j}^{\prime}>0$ if $G_{j} \in \mathcal{S}, b_{j}, b_{j}^{\prime} \geq 0$ if $G_{j}$ is an ergodic measure. The pair of holomorphic quadratic differentials $q, q^{\prime}$ or measured foliations $H(q), H\left(q^{\prime}\right)$ is called absolutely continuous if $q, q^{\prime}$ are topologically equivalent and for the representations $H(q)=$ $\sum_{j=1}^{k} b_{j} G_{j}, H\left(q^{\prime}\right)=\sum_{j=1}^{k} b_{j}^{\prime} G_{j}$, the set of subscripts of non-zero coefficients $b_{j}$ of $H(q)$ and the one of $H\left(q^{\prime}\right)$ coincide. In this situation, we can set $b_{j}, b_{j}^{\prime}>0$ for any $j=1, \cdots, k$.

Remark. If two holomorphic quadratic differentials $q, q^{\prime}$ are Jenkins-Strebel and topologically equivalent, then they are absolutely continuous. 
2.5. Teichmüller geodesic rays. Let $q$ be a unit norm holomorphic quadratic differential on $X$. A quasiconformal mapping $f$ on $X$ is called a Teichmüller mapping for $q$ if $f$ has the Beltrami coefficient $-\frac{K(f)-1}{K(f)+1} \frac{\bar{q}}{|q|}$. The existence and uniqueness for Teichmüller mappings are the following.

Theorem 2.2 (Teichmüller's existence theorem). For any quasiconformal mapping $g: X \rightarrow Y$, there is a Teichmüller mapping $f$ which is homotopic to $g$.

Theorem 2.3 (Teichmüller's uniqueness theorem). For any quasiconformal mapping $g: X \rightarrow Y$ which is homotopic to the Teichmüller mapping $f$, the inequality $K(f) \leq K(g)$ holds and the equality holds if and only if $f=g$.

These statements are called Teichmüller's theorem. (We refer to [T92] for details.) Therefore, a Teichmüller mapping is attained for the infimum of the definition of the Teichmüller distance, i.e., for any $p_{1}=\left[Y_{1}, f_{1}\right], p_{2}=\left[Y_{2}, f_{2}\right] \in T(X)$, there is the Teichmüller mapping $h: Y_{1} \rightarrow Y_{2}$ which is homotopic to $f_{2} \circ f_{1}^{-1}$ such that $d_{T(X)}\left(p_{1}, p_{2}\right)=\frac{1}{2} \log K(h)$.

For any $p=[Y, f] \in T(X)$, let $q$ be a unit norm holomorphic quadratic differential on $Y$ and let $z=x+i y$ be any $q$-coordinate. For any $t \geq 0$, let $Y_{t}$ be a Riemann surface determined by each local coordinate $z_{t}=e^{-t} x+i e^{t} y$ and let $g_{t}: Y \rightarrow Y_{t}$ be a Teichmüller mapping which is determined by $z \mapsto z_{t}$. We assume that $Y_{0}=Y, g_{0}=i d_{Y}$. We set $r(t)=\left[Y_{t}, g_{t} \circ f\right]$; then by Teichmüller's theorem, this mapping $r: \mathbb{R}_{>0} \rightarrow T(X)$ is an isometry and is called the Teichmüller geodesic ray on $T(X)$ starting at $p$ and having the initial holomorphic quadratic differential $q$. If the holomorphic quadratic differential $q$ is Jenkins-Strebel, the ray $r$ is called a Jenkins-Strebel ray.

Definition 2.4. Let $r, r^{\prime}$ be Teichmüller geodesic rays on $T(X)$ starting at $r(0)=$ $[Y, f], r^{\prime}(0)=\left[Y^{\prime}, f^{\prime}\right]$ and having initial unit norm holomorphic quadratic differentials $q, q^{\prime}$ on $Y, Y^{\prime}$, respectively. We denote by $H(q) \in \mathcal{M F}(Y), H\left(q^{\prime}\right) \in$ $\mathcal{M F}\left(Y^{\prime}\right)$ the measured foliations corresponding to $q, q^{\prime}$, respectively. We suppose that $f_{*}^{-1}(H(q)), f_{*}^{\prime-1}\left(H\left(q^{\prime}\right)\right) \in \mathcal{M F}(X)$ are absolutely continuous, then the measured foliations are written as $f_{*}^{-1}(H(q))=\sum_{j=1}^{k} b_{j} G_{j}, f_{*}^{\prime-1}\left(H\left(q^{\prime}\right)\right)=\sum_{j=1}^{k} b_{j}^{\prime} G_{j}$, $H(q)=\sum_{j=1}^{k} b_{j} f_{*}\left(G_{j}\right)$ and $H\left(q^{\prime}\right)=\sum_{j=1}^{k} b_{j}^{\prime} f_{*}^{\prime}\left(G_{j}\right)$ where $b_{1}, \cdots, b_{k}, b_{1}^{\prime}, \cdots, b_{k}^{\prime}$ are positive real numbers and $G_{j}$ is a simple closed curve or an ergodic measure for any $j=1, \cdots, k$ such that these are projectively distinct and pairwise having zero intersection number. We set $m_{j}=\frac{b_{j}}{i\left(f_{*}\left(G_{j}\right), V(q)\right)}, m_{j}^{\prime}=\frac{b_{j}^{\prime}}{i\left(f_{*}^{\prime}\left(G_{j}\right), V\left(q^{\prime}\right)\right)}$ for any $j=1, \cdots, k$. In this situation, the given rays $r, r^{\prime}$ are called modularly equivalent if there is $\lambda>0$ such that $m_{j}^{\prime}=\lambda m_{j}$ for any $j=1, \cdots, k$.

Definition 2.5. The pair of Teichmüller geodesic rays $r, r^{\prime}$ on $T(X)$ are called bounded if there is $M>0$ such that $d_{T(X)}\left(r(t), r^{\prime}(t)\right)<M$ for any $t \geq 0$, divergent if $d_{T(X)}\left(r(t), r^{\prime}(t)\right) \rightarrow+\infty$ as $t \rightarrow \infty$, and asymptotic if there is a choice of initial points $r(0), r^{\prime}(0)$ such that $d_{T(X)}\left(r(t), r^{\prime}(t)\right) \rightarrow 0$ as $t \rightarrow \infty$; in other words, for the given rays $r(t), r^{\prime}(t)$, there is $\alpha \in \mathbb{R}$ such that $d_{T(X)}\left(r(t), r^{\prime}(t+\alpha)\right) \rightarrow 0$ as $t \rightarrow \infty$.

2.6. The end point of a Jenkins-Strebel ray. In this section, we refer to Abi77, HS07] and [T92.

Definition 2.6 (Riemann surfaces with nodes). A connected Hausdorff space $R$ is called a Riemann surface of type $(g, n)$ with nodes if $R$ satisfies the following conditions: 
(1) Any $p \in R$ has a neighborhood which is homeomorphic to the unit disk $\mathbb{D}$ or the set $\left\{\left(z_{1}, z_{2}\right) \in \mathbb{C}^{2}|| z_{1}|<1,| z_{2} \mid<1, z_{1} \cdot z_{2}=0\right\}$. (In the latter case, $p$ is called a node of $R$. We allow $R$ to have finitely many nodes.)

(2) Let $p_{1}, \cdots, p_{k}$ be nodes of $R$. We denote by $R_{1}, \cdots, R_{r}$ the connected components of $R-\left\{p_{1}, \cdots, p_{k}\right\}$. For any $i=1, \cdots, r$, each $R_{i}$ is of type $\left(g_{i}, n_{i}\right)$ which satisfies $2 g_{i}-2+n_{i}>0, n=\sum_{i=1}^{r} n_{i}-2 k$ and $g=\sum_{i=1}^{r} g_{i}-$ $r+k+1$.

Remark. Condition (2) means that we get a Riemann surface of type $(g, n)$ without nodes by opening each node of $R$. All Riemann surfaces of type $(g, n)$ without nodes are included to this definition.

Definition 2.7 (Augmented Teichmüller spaces). Let $X$ be a Riemann surface of type $(g, n)$ without nodes which satisfies $3 g-3+n>0$. The augmented Teichmüller space $\hat{T}(X)$ of $X$ is the set of all equivalence classes $[R, f]$ of pairs of a Riemann surface $R$ of type $(g, n)$ with nodes and a deformation $f: X \rightarrow R$ which is a mapping such that it contracts some disjoint loops on $X$ to points (the nodes of $R$ ) and is a homeomorphism except on the loops. Two pairs $\left(R_{1}, f_{1}\right)$ and $\left(R_{2}, f_{2}\right)$ are equivalent if there is a biholomorphic mapping $h: R_{1} \rightarrow R_{2}$ such that $f_{2}$ is homotopic to $h \circ f_{1}$. Here, for Riemann surfaces with nodes $R$ and $S$, a homeomorphism $f: R \rightarrow S$ is called biholomorphic if each restricted mapping of $f$ which maps a component of $R-\{$ nodes of $R$ \} onto a component of $S-$ nodes of $S$ \} is biholomorphic. A topology on $\hat{T}(X)$ is defined by the following neighborhoods. For any compact neighborhood $V$ of the set of nodes in $R$ and any $\varepsilon>0$, a neighborhood $U_{V, \varepsilon}$ of a point $[R, f]$ is defined by

$U_{V, \varepsilon}=\{[S, g] \in \hat{T}(X) \mid$ there is a deformation $h: S \rightarrow R$ which is

$(1+\varepsilon)$-quasiconformal on $h^{-1}(R-V)$ such that $f$ is homotopic to $h \circ g$ \}.

Let $r$ be the Jenkins-Strebel ray on $T(X)$ starting at $r(0)=[Y, f]$ and having an initial unit norm Jenkins-Strebel differential $q$ on $Y$. We set $r(t)=\left[Y_{t}, g_{t} \circ f\right]$ for any $t \geq 0$. We consider the end point of $r$ in $\hat{T}(X)$. We denote by $H(q)=$ $\sum_{j=1}^{k} b_{j} \gamma_{j}$ the measured foliation constructed by $q$. The surface $Y-\Gamma_{q}$ consists of annuli corresponding to $\gamma_{1}, \cdots, \gamma_{k}$, and let $m_{1}, \cdots, m_{k}$ be moduli of these annuli, respectively. By $q$-coordinates, the annuli are represented by Euclidean cylinders. For each cylinder, we use polar coordinates and describe it as two round annuli:

$$
A_{j}^{1}(0)=A_{j}^{2}(0)=\left\{w \in \mathbb{C}\left|\exp \left(-m_{j} \pi\right) \leq\right| w \mid<1\right\} .
$$

We glue $A_{j}^{1}(0)$ and $A_{j}^{2}(0)$ at the line of these inner boundary $|w|=\exp \left(-m_{j} \pi\right)$ by the mapping $w \mapsto \frac{\exp \left(-2 m_{j} \pi\right)}{w}$ and denote by $A_{j}(0)$ the resulting surface. We obtain the base surface $Y$ after gluing $\overline{A_{1}(0)}, \cdots, \overline{A_{k}(0)}$ by the gluing mappings which are determined by $q$. The Teichmüller mapping $g_{t}: Y \rightarrow Y_{t}$ is represented by $w=r e^{i \theta} \mapsto r^{\exp (2 t)} e^{i \theta}$ for any $t \geq 0$. We fix any $t \geq 0$ and define

$$
A_{j}^{1}(t)=A_{j}^{2}(t)=\left\{w \in \mathbb{C}\left|\exp \left(-e^{2 t} m_{j} \pi\right) \leq\right| w \mid<1\right\} .
$$

The gluing mapping of $A_{j}^{1}(t)$ onto $A_{j}^{2}(t)$ is $w \mapsto \frac{\exp \left(-2 e^{2 t} m_{j} \pi\right)}{w}$ in their inner boundary $|w|=\exp \left(-e^{2 t} m_{j} \pi\right)$, and we denote by $A_{j}(t)$ the resulting surface. The surface $Y_{t}$ is obtained by $\overline{A_{1}(t)}, \cdots, \overline{A_{k}(t)}$ with the same gluing mappings as in the case of $t=0$.

Remark. We notice that the modulus of $A_{j}(t)$ is equal to $e^{2 t} m_{j}$ for any $t \geq 0$ and any $j=1, \cdots, k$. 
We consider the limit of $A_{j}(t)$ as $t \rightarrow \infty$ for any $j=1, \cdots, k$. We set $A_{j}^{1}(\infty)=$ $A_{j}^{2}(\infty)=\{w \in \mathbb{C}|0<| w \mid<1\}$, and denote by $\{p t\}$ the set which consists of an arbitrary point. The disjoint union $A_{j}(\infty)=A_{j}^{1}(\infty) \cup A_{j}^{2}(\infty) \cup\{p t\}$ becomes a complex cone by the following chart:

$$
\begin{gathered}
\Phi: A_{j}(\infty) \rightarrow\left\{\left(w^{1}, w^{2}\right) \in \mathbb{C}^{2}|| w^{1}|<1,| w^{2} \mid<1, w^{1} \cdot w^{2}=0\right\} \\
\left.\Phi\right|_{A_{j}^{1}(\infty)}(w)=(w, 0),\left.\Phi\right|_{A_{j}^{2}(\infty)}(w)=(0, w), \Phi(p t)=(0,0)
\end{gathered}
$$

for any $j=1, \cdots, k$. We denote by $Y_{\infty}$ the surface constructed by $\overline{A_{1}(\infty)}, \cdots$, $\overline{A_{k}(\infty)}$ with the same gluing mappings as in the case of $t=0$. The mapping $g_{\infty}: Y \rightarrow Y_{\infty}$ is constructed by the mapping of $A_{j}^{l}(0)$ onto $A_{j}^{l}(\infty) \cup\{p t\}$ by $w=$ $r e^{i \theta} \mapsto h_{j}(r) e^{i \theta}$ where $h_{j}:\left[\exp \left(-m_{j} \pi\right), 1\right) \rightarrow[0,1)$ is an arbitrary monotonously increasing diffeomorphism for any $j=1, \cdots, k$ and $l=1,2$. The homotopy class of $g_{\infty}$ is independent of the choices of $h_{j}$ for any $j=1, \cdots, k$. Then, we obtain $\left[Y_{\infty}, g_{\infty} \circ f\right]$ in $\hat{T}(X)$ and denote it by $r(\infty)$. From the definition of a neighborhood of $\left[Y_{\infty}, g_{\infty} \circ f\right]$, the following proposition holds.

Proposition 2.8 ([HS07]). The Jenkins-Strebel ray $r(t)=\left[Y_{t}, g_{t} \circ f\right]$ on $T(X)$ starting at $r(0)=[Y, f]$ and having the initial unit norm Jenkins-Strebel differential $q$ on $Y$ converges to a point $r(\infty)=\left[Y_{\infty}, g_{\infty} \circ f\right]$ in $\hat{T}(X)$.

Remark. We can reconstruct the surface $Y_{t}$ from $Y_{\infty}$ for any $t \geq 0$. First, for any $j=1, \cdots, k$ and $l=1,2$, we remove the punctured disk $\{w \in \mathbb{C}|0<| w \mid<$ $\left.\exp \left(-e^{2 t} m_{j} \pi\right)\right\}$ from $A_{j}^{l}(\infty)$ of $Y_{\infty}$. Then, the interior of each resulting annulus is biholomorphic to the interior of $A_{j}^{l}(t)$. We obtain $Y_{t}$ by gluing $\left\{{\overline{A_{j}^{l}(t)}}_{j=1, \cdots, k}^{l=1,2}\right.$ with the suitable gluing mappings.

2.7. Extremal lengths. Let $\rho$ be a locally $L^{1}$-measurable conformal metric on a Riemann surface $Y$, i.e., it is represented by the form $\rho=\rho(z)|d z|$ on any local coordinate $z$ of $Y$ where $\rho(z)$ is a non-negative measurable function of $z$ such that the equation $\rho(z)|d z|=\rho(w)|d w|$ holds for any local coordinates $z, w$ on a common neighborhood of $Y$. For any $\gamma \in \mathcal{S}$, we define the $\rho$-length of $\gamma$ on $Y$ and the $\rho$-area of $Y$ by

$$
\begin{gathered}
l_{\rho}(\gamma)=\inf _{\gamma^{\prime} \in \gamma} \int_{\gamma^{\prime}} \rho(z)|d z|, \\
A_{\rho}=\iint_{Y} \rho(z)^{2} d x d y,
\end{gathered}
$$

respectively. The extremal length $\operatorname{Ext}_{Y}(\gamma)$ of $\gamma$ on $Y$ is defined by the following:

$$
\operatorname{Ext}_{Y}(\gamma)=\sup _{\rho} \frac{l_{\rho}(\gamma)^{2}}{A_{\rho}}
$$

where $\rho$ ranges over all locally $L^{1}$-measurable conformal metrics on $Y$ such that $0<A_{\rho}<\infty$.

For any $p=[Y, f] \in T(X)$ and any $\alpha \in \mathcal{S}$ on $X$, we define

$$
\operatorname{Ext}_{p}(\alpha)=\operatorname{Ext}_{Y}\left(f_{*}(\alpha)\right) \text {. }
$$

Theorem 2.9 ([Ker80]). For any Riemann surface $Y$, each extremal length on $Y$ extends continuously on $\mathcal{M F}(Y)$ such that the equation $\operatorname{Ext}_{Y}(t \nu)=t^{2} \operatorname{Ext}_{Y}(\nu)$ holds for any $t \geq 0$ and any $\nu \in \mathcal{M F}(Y)$.

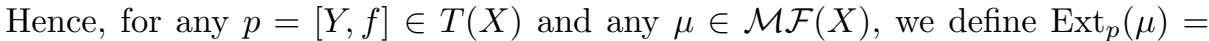
$\operatorname{Ext}_{Y}\left(f_{*}(\mu)\right)$ and the equation $\operatorname{Ext}_{p}(t \mu)=t^{2} \operatorname{Ext}_{p}(\mu)$ holds for any $t \geq 0$. We notice 
that for any $p \in T(X)$ and any $\mu \in \mathcal{M F}(X)-\{0\}$, the extremal length $\operatorname{Ext}_{p}(\mu)$ is positive. We set $\mathcal{M F}(X)^{*}=\mathcal{M F}(X)-\{0\}$.

Theorem 2.10 (Kerckhoff's formula for the Teichmüller distance [Ker80]). For any $p_{1}, p_{2} \in T(X)$, the Teichmüller distance between $p_{1}$ and $p_{2}$ is represented by

$$
d_{T}\left(p_{1}, p_{2}\right)=\frac{1}{2} \log \sup _{\mu \in \mathcal{M} \mathcal{F}(X) *} \frac{\operatorname{Ext}_{p_{2}}(\mu)}{\operatorname{Ext}_{p_{1}}(\mu)} .
$$

Kerckhoff's formula is useful for finding lower bounds of the Teichmüller distance.

\section{Proof of Theorem}

In this section, we prove Theorem 1.1 which is our main theorem. Let $r, r^{\prime}$ be Jenkins-Strebel rays on $T(X)$ starting at $r(0)=[Y, f], r(0)^{\prime}=\left[Y^{\prime}, f^{\prime}\right]$ and having initial unit norm Jenkins-Strebel differentials $q, q^{\prime}$ on $Y, Y^{\prime}$, respectively. Let $r(\infty), r^{\prime}(\infty)$ be the end points of $r, r^{\prime}$ in the augmented Teichmüller space $\hat{T}(X)$, respectively. We denote by $H(q) \in \mathcal{M F}(Y), H\left(q^{\prime}\right) \in \mathcal{M F}\left(Y^{\prime}\right)$ the corresponding measured foliations and suppose that the measured foliations $f_{*}^{-1}(H(q))$, $f_{*}^{\prime-1}\left(H\left(q^{\prime}\right)\right) \in \mathcal{M F}(X)$ are topologically equivalent. In this situation, we can write $f_{*}^{-1}(H(q))=\sum_{j=1}^{k} b_{j} \gamma_{j}, f_{*}^{\prime-1}\left(H\left(q^{\prime}\right)\right)=\sum_{j=1}^{k} b_{j}^{\prime} \gamma_{j}$ where $b_{1}, \cdots, b_{k}, b_{1}^{\prime}, \cdots, b_{k}^{\prime}$ are positive real numbers and $\gamma_{1}, \cdots, \gamma_{k}$ are distinct and pairwise having zero intersection number. We denote by $m_{j}=\frac{b_{j}}{i\left(f_{*}\left(\gamma_{j}\right), V(q)\right)}, m_{j}^{\prime}=\frac{b_{j}^{\prime}}{i\left(f_{*}^{\prime}\left(\gamma_{j}\right), V\left(q^{\prime}\right)\right)}$ the moduli of the annuli of $H(q), H\left(q^{\prime}\right)$ corresponding to $f_{*}\left(\gamma_{j}\right), f_{*}^{\prime}\left(\gamma_{j}\right)$ for any $j=1, \cdots, k$.

Theorem 1.1. If $r(\infty)=r^{\prime}(\infty)$, then

$$
\lim _{t \rightarrow \infty} d_{T(X)}\left(r(t), r^{\prime}(t)\right)=\frac{1}{2} \log \max _{j=1, \cdots, k}\left\{\frac{m_{j}^{\prime}}{m_{j}}, \frac{m_{j}}{m_{j}^{\prime}}\right\} .
$$

We represent the Jenkins-Strebel rays by $r(t)=\left[Y_{t}, g_{t} \circ f\right], r^{\prime}(t)=\left[Y_{t}^{\prime}, g_{t}^{\prime} \circ f^{\prime}\right]$ for any $t \geq 0$, and their end points by $r(\infty)=\left[Y_{\infty}, g_{\infty} \circ f\right], r^{\prime}(\infty)=\left[Y_{\infty}^{\prime}, g_{\infty}^{\prime} \circ f^{\prime}\right]$, respectively. Since the measured foliations $f_{*}^{-1}(H(q)), f_{*}^{\prime-1}\left(H\left(q^{\prime}\right)\right)$ are topologically equivalent, there is a homeomorphism $\alpha: X-\Gamma_{f_{*}^{-1}(H(q))} \rightarrow X-\Gamma_{f_{*}^{\prime-1}\left(H\left(q^{\prime}\right)\right)}$ which is homotopic to the identity such that the leaves of $f_{*}^{-1}(H(q))$ are mapped to the leaves of $f_{*}^{\prime-1}\left(H\left(q^{\prime}\right)\right)$. Then, the mapping $f^{\prime} \circ \alpha \circ f^{-1}$ which is homotopic to $f^{\prime} \circ f^{-1}$ maps the leaves of $H(q)$ to the leaves of $H\left(q^{\prime}\right)$. We denote by $A_{j}(0)$, $A_{j}^{\prime}(0)$ the annuli corresponding to $f_{*}\left(\gamma_{j}\right), f_{*}^{\prime}\left(\gamma_{j}\right)$, respectively, for any $j=1, \cdots, k$. They are the same as in $\S 2.6$. Then, the equations $f_{*}^{\prime} \circ \alpha_{*} \circ f_{*}^{-1}\left(f_{*}\left(\gamma_{j}\right)\right)=f_{*}^{\prime}\left(\gamma_{j}\right)$, $f^{\prime} \circ \alpha \circ f^{-1}\left(A_{j}(0)\right)=A_{j}^{\prime}(0)$ hold for any $j=1, \cdots, k$. For any $t \geq 0$ and any $j=1, \cdots, k$, the annuli $A_{j}(t), A_{j}^{\prime}(t)$ can be determined the same as in $\$ 2.6$ and we can see that $\left(g_{t}^{\prime} \circ f^{\prime}\right) \circ \alpha \circ\left(g_{t} \circ f\right)^{-1}\left(A_{j}(t)\right)=A_{j}^{\prime}(t)$.

In order to prove the equation of Theorem 1.1, we consider the upper and lower estimates of the limit supremum and limit infimum of the distance between the rays, respectively.

3.1. Upper estimate. First, we show that

$$
\limsup _{t \rightarrow \infty} d_{T(X)}\left(r(t), r^{\prime}(t)\right) \leq \frac{1}{2} \log \max _{j=1, \cdots, k}\left\{\frac{m_{j}^{\prime}}{m_{j}}, \frac{m_{j}}{m_{j}^{\prime}}\right\} .
$$

The idea of the following lemma comes from Gup11. 
Lemma 3.1. Let us choose $0<\varepsilon<1$ arbitrarily. Then, for any sufficiently large $t$, there is a quasiconformal mapping $F_{t}: Y_{t} \rightarrow Y_{t}^{\prime}$ which is homotopic to $\left(g_{t}^{\prime} \circ f^{\prime}\right) \circ\left(g_{t} \circ f\right)^{-1}$ such that the inequality $\lim _{t \rightarrow \infty} K\left(F_{t}\right)<\max _{j=1, \cdots, k}\left\{\frac{m_{j}^{\prime}}{m_{j}}, \frac{m_{j}}{m_{j}^{\prime}}\right\}+\varepsilon$ holds.

Proof. We set $M_{j}=\frac{m_{j}^{\prime}}{m_{j}}$ for any $j=1, \cdots, k$. By the equation $r(\infty)=r^{\prime}(\infty)$, there exists a biholomorphic mapping $h: Y_{\infty} \rightarrow Y_{\infty}^{\prime}$ such that $h \circ g_{\infty} \circ f$ is homotopic to $g_{\infty}^{\prime} \circ f^{\prime}$. From $\S 2.6$, we can write

$$
\begin{gathered}
Y_{\infty}=\bigcup_{j=1}^{k} \overline{A_{j}^{1}(\infty)} \cup \overline{A_{j}^{2}(\infty)}, \\
Y_{\infty}^{\prime}=\bigcup_{j=1}^{k} \overline{A_{j}^{\prime 1}(\infty)} \cup \overline{A_{j}^{\prime 2}(\infty)},
\end{gathered}
$$

where $A_{j}^{l}(\infty), A_{j}^{\prime l}(\infty)$ are the punctured disks $\mathbb{D}^{*}=\{z \in \mathbb{C}|0<| z \mid<1\}$ for any $j=1, \cdots, k$ and $l=1,2$. Now, we fix any $j=1, \cdots, k$ and $l=1,2$. We set $h_{j}^{l}=\left.h\right|_{A_{j}^{l}(\infty)}: A_{j}^{l}(\infty) \rightarrow h\left(A_{j}^{l}(\infty)\right) \subset Y_{\infty}^{\prime}$. Since $h$ is a biholomorphic mapping, we can set $h_{j}^{l}(0)=0$ and $\left.\frac{d h_{j}^{l}(z)}{d z}\right|_{z=0} \neq 0$. We describe $h_{j}^{l}(z)=c_{j}^{l} z+c_{j, 2}^{l} z^{2}+\cdots=$ $c_{j}^{l} z+\psi_{j}^{l}(z)$ where $c_{j}^{l} \neq 0,-\pi<\arg c_{j}^{1} \leq \pi$ and $-\pi \leq \arg c_{j}^{2}<\pi$. For any $t \geq 0$, we set $\delta_{j}(t)=\exp \left(-e^{2 t} m_{j} \pi\right), \delta_{j}^{\prime}(t)=\exp \left(-e^{2 t} m_{j}^{\prime} \pi\right)$, then $\delta_{j}^{\prime}(t)=\delta_{j}(t)^{M_{j}}$. After this, we assume that $A_{j}^{l}(t)=\mathbb{D}^{*}-\mathbb{D}_{\delta_{j}(t)}=\left\{z \in \mathbb{C}\left|\delta_{j}(t) \leq\right| z \mid<1\right\}$ and $A_{j}^{\prime}(t)=\mathbb{D}^{*}-\mathbb{D}_{\delta_{j}^{\prime}(t)}=\left\{z \in \mathbb{C}\left|\delta_{j}^{\prime}(t) \leq\right| z \mid<1\right\}$ for any $t \geq 0$. For sufficiently large $t$, we construct a quasiconformal mapping $F_{j, t}^{l}: \mathbb{D}^{*}-\mathbb{D}_{\delta_{j}(t)} \rightarrow h_{j}^{l}\left(\mathbb{D}^{*}\right)-\mathbb{D}_{\delta_{j}^{\prime}(t)}$. We consider the following three cases (1), (2) and (3).

(1) In the case of $M_{j}>1$, we take $X_{j}$ as

$$
X_{j}<\frac{\log \frac{\varepsilon}{M_{j}+\varepsilon-1}}{\log M_{j}}<0 .
$$

This is equivalent to

$$
M_{j}^{X_{j}}<\frac{\varepsilon}{M_{j}+\varepsilon-1}<1
$$

and

$$
\frac{M_{j}-M_{j}^{X_{j}}}{1-M_{j}^{X_{j}}}<M_{j}+\varepsilon
$$

We take sufficiently large $t$ such that the inequality $\delta_{j}(t)^{M_{j}}<\left|c_{j}^{l}\right| \delta_{j}(t)^{M_{j}^{X_{j}}}$ holds. We set $\Delta_{j}(t)=\delta_{j}(t)^{M_{j}^{X_{j}}}$. We construct $F_{j, t}^{l}$ by the following:

$$
F_{j, t}^{l}(z)= \begin{cases}P_{j, t}^{l}(z) & \left(\delta_{j}(t) \leq|z| \leq \Delta_{j}(t)\right) \\ Q_{j, t}^{l}(z) & \left(\Delta_{j}(t) \leq|z| \leq 2 \Delta_{j}(t)\right) \\ h_{j}^{l}(z) & \left(2 \Delta_{j}(t) \leq|z|<1\right)\end{cases}
$$

(i) In $\delta_{j}(t) \leq|z| \leq \Delta_{j}(t)$, we set 


$$
P_{j, t}^{l}(z)=\Delta_{j}(t)^{\frac{1-M_{j}}{1-M_{j}^{X}}} \cdot c_{j}^{\frac{1}{1-M_{j}^{X}}+\frac{\log |z|}{\log \Delta_{j}(t)-\log \delta_{j}(t)}} \cdot|z|^{-\frac{1-M_{j}}{1-M_{j}^{X}}} \cdot z
$$

which satisfies $P_{j, t}^{l}(z)=\delta_{j}(t)^{M_{j}-1} \cdot z$ on $|z|=\delta_{j}(t), P_{j, t}^{l}(z)=c_{j}^{l} z$ on $|z|=\Delta_{j}(t)$. The mapping $P_{j, t}^{l}$ is conjugate to a one-to-one affine mapping by $\log z$. Then, $P_{j, t}^{l}$ is a quasiconformal mapping, and its dilatation is the following:

$$
K\left(P_{j, t}^{l}\right)=\frac{\left|\frac{\log c_{j}^{l}}{2\left(\log \Delta_{j}(t)-\log \delta_{j}(t)\right)}+\frac{\alpha_{j}}{2}+1\right|+\left|\frac{\log c_{j}^{l}}{2\left(\log \Delta_{j}(t)-\log \delta_{j}(t)\right)}+\frac{\alpha_{j}}{2}\right|}{\left|\frac{\log c_{j}^{l}}{2\left(\log \Delta_{j}(t)-\log \delta_{j}(t)\right)}+\frac{\alpha_{j}}{2}+1\right|-\left|\frac{\log c_{j}^{l}}{2\left(\log \Delta_{j}(t)-\log \delta_{j}(t)\right)}+\frac{\alpha_{j}}{2}\right|},
$$

where

$$
\alpha_{j}=-\frac{1-M_{j}}{1-M_{j}^{X_{j}}} .
$$

We see that $\log \Delta_{j}(t)-\log \delta_{j}(t)=\left(M_{j}^{X_{j}}-1\right) \log \delta_{j}(t) \rightarrow+\infty$ and

$$
K\left(P_{j, t}^{l}\right) \rightarrow \frac{M_{j}-M_{j}^{X_{j}}}{1-M_{j}^{X_{j}}}<M_{j}+\varepsilon
$$

as $t \rightarrow \infty$.

(ii) In $\Delta_{j}(t) \leq|z| \leq 2 \Delta_{j}(t)$, we set

$$
Q_{j, t}^{l}(z)=c_{j}^{l} z+\phi_{\Delta_{j}(t)}(|z|) \psi_{j}^{l}(z),
$$

where $\phi_{\Delta_{j}(t)}:\left[\Delta_{j}(t), 2 \Delta_{j}(t)\right] \rightarrow[0,1]$ is defined by

$$
\phi_{\Delta_{j}(t)}(|z|)=\frac{|z|}{\Delta_{j}(t)}-1
$$

This function satisfies $Q_{j, t}^{l}(z)=c_{j}^{l} z$ on $|z|=\Delta_{j}(t), Q_{j, t}^{l}(z)=h_{j}^{l}(z)$ on $|z|=2 \Delta_{j}(t)$. We consider the partial derivatives of $Q_{j, t}^{l}$,

$$
\begin{gathered}
\partial_{\bar{z}} Q_{j, t}^{l}=\frac{1}{2 \Delta_{j}(t)} z^{\frac{1}{2}} \bar{z}^{-\frac{1}{2}} \psi_{j}^{l}(z) \\
\partial_{z} Q_{j, t}^{l}=c_{j}^{l}+\frac{1}{2 \Delta_{j}(t)} z^{-\frac{1}{2}} \bar{z}^{\frac{1}{2}} \psi_{j}^{l}(z)+\phi_{\Delta(t)}(|z|) \frac{d \psi_{j}^{l}(z)}{d z} .
\end{gathered}
$$

These partial derivatives are continuous in $\Delta_{j}(t) \leq|z| \leq 2 \Delta_{j}(t)$. There is $C>0$ such that $\left|\psi_{j}^{l}(z)\right| \leq C \Delta_{j}(t)^{2}$ for sufficiently large $t$. We see that

$$
\left|\frac{1}{2 \Delta_{j}(t)} z^{\frac{1}{2}} \bar{z}^{-\frac{1}{2}} \psi_{j}^{l}(z)\right|=\left|\frac{1}{2 \Delta_{j}(t)} z^{-\frac{1}{2}} \bar{z}^{\frac{1}{2}} \psi_{j}^{l}(z)\right|=\frac{\left|\psi_{j}^{l}(z)\right|}{2 \Delta_{j}(t)} \leq \frac{C \Delta_{j}(t)}{2} \rightarrow 0
$$

as $t \rightarrow \infty$. Then, $\left|\partial_{\bar{z}} Q_{j, t}^{l}\right| \rightarrow 0,\left|\partial_{z} Q_{j, t}^{l}\right| \rightarrow\left|c_{j}^{l}\right| \neq 0$ as $t \rightarrow \infty$. For sufficiently large $t$, Jac $Q_{j, t}^{l}=\left|\partial_{z} Q_{j, t}^{l}\right|^{2}-\left|\partial_{\bar{z}} Q_{j, t}^{l}\right|^{2}>0$. Hence, $Q_{j, t}^{l}$ is a local $C^{1}$-diffeomorphism. We denote by $D$ the closed set whose boundary consists of two components $Q_{j, t}^{l}(\{|z|=$ $\left.\left.\Delta_{j}(t)\right\}\right)=\left\{|w|=\left|c_{j}^{l}\right| \Delta_{j}(t)\right\}$ and $Q_{j, t}^{l}\left(\left\{|z|=2 \Delta_{j}(t)\right\}\right)=h_{j}^{l}\left(\left\{|z|=2 \Delta_{j}(t)\right\}\right)$, and its fundamental group is $\pi_{1}(D)=\mathbb{Z}$. The equation $Q_{j, t}^{l}\left(\left\{\Delta_{j}(t) \leq|z| \leq 2 \Delta_{j}(t)\right\}\right)=D$ holds because $Q_{j, t}^{l}$ is a local $C^{1}$-diffeomorphism with the above boundary conditions. We regard the mapping $Q_{j, t}^{l}:\left\{\Delta_{j}(t) \leq|z| \leq 2 \Delta_{j}(t)\right\} \rightarrow D$ as a covering. 
Let $Q_{j, t *}^{l}: \pi_{1}\left(\left\{\Delta_{j}(t) \leq|z| \leq 2 \Delta_{j}(t)\right\}\right) \rightarrow \pi_{1}(D)$ be the group homomorphism induced by $Q_{j, t}^{l}$. We see that $Q_{j, t *}^{l}\left(\pi_{1}\left(\left\{\Delta_{j}(t) \leq|z| \leq 2 \Delta_{j}(t)\right\}\right)\right)=\mathbb{Z} \triangleleft \pi_{1}(D)$ because $Q_{j, t}^{l}(z)=c_{j}^{l} z$ on $|z|=\Delta_{j}(t)$. Then, the covering $Q_{j, t}^{l}$ is a regular covering, and its covering transformation group is $\mathbb{Z} / \mathbb{Z}=1$. Therefore, $Q_{j, t}^{l}$ is a $C^{1}$-diffeomorphism. By the partial derivatives of $Q_{j, t}^{l}$, for sufficiently large $t$, it is a quasiconformal mapping such that its dilatation holds $K\left(Q_{j, t}^{l}\right) \rightarrow 1$ as $t \rightarrow \infty$.

(iii) In $2 \Delta_{j}(t) \leq|z|<1, F_{j, t}^{l}(z)=h_{j}^{l}(z)$ and $K\left(h_{j}^{l}\right)=1$.

Therefore, for sufficiently large $t$, we obtain the quasiconformal mapping $F_{j, t}^{l}$ such that

$$
K\left(F_{j, t}^{l}\right)=\max \left\{K\left(P_{j, t}^{l}\right), K\left(Q_{j, t}^{l}\right)\right\} \rightarrow \frac{M_{j}-M_{j}^{X_{j}}}{1-M_{j}^{X_{j}}}<M_{j}+\varepsilon
$$

as $t \rightarrow \infty$.

(2) In the case of $M_{j}<1$, we take $X_{j}$ as

$$
X_{j}>\frac{\log \frac{M_{j} \varepsilon}{\frac{1}{M_{j}}-1+\varepsilon}}{\log M_{j}}>2 \text {. }
$$

This is equivalent to

$$
M_{j}^{X_{j}}<\frac{M_{j} \varepsilon}{\frac{1}{M_{j}}-1+\varepsilon}<M_{j}^{2}
$$

and

$$
\frac{1-M_{j}^{X_{j}}}{M_{j}-M_{j}^{X_{j}}}<\frac{1}{M_{j}}+\varepsilon
$$

We take sufficiently large $t$ such that the inequality $\delta_{j}(t)^{M_{j}}<\left|c_{j}^{l}\right| \delta_{j}(t)^{M_{j}^{X_{j}}}$ holds. We also set $\Delta_{j}(t)=\delta_{j}(t)^{M_{j}^{X_{j}}}$, and also construct $F_{j, t}^{l}$ by the following:

$$
F_{j, t}^{l}(z)= \begin{cases}P_{j, t}^{l}(z) & \left(\delta_{j}(t) \leq|z| \leq \Delta_{j}(t)\right), \\ Q_{j, t}^{l}(z) & \left(\Delta_{j}(t) \leq|z| \leq 2 \Delta_{j}(t)\right), \\ h_{j}^{l}(z) & \left(2 \Delta_{j}(t) \leq|z|<1\right) .\end{cases}
$$

The functions $P_{j, t}^{l}, Q_{j, t}^{l}$ have the same notations as in the case of (1). The difference is only the dilatation of $P_{j, t}^{l}$. In this case, we see that

$$
K\left(P_{j, t}^{l}\right) \rightarrow \frac{1-M_{j}^{X_{j}}}{M_{j}-M_{j}^{X_{j}}}<\frac{1}{M_{j}}+\varepsilon
$$

as $t \rightarrow \infty$. Similarly, as in the case of (1), for sufficiently large $t$, we obtain the quasiconformal mapping $F_{j, t}^{l}$ such that

$$
K\left(F_{j, t}^{l}\right)=\max \left\{K\left(P_{j, t}^{l}\right), K\left(Q_{j, t}^{l}\right)\right\} \rightarrow \frac{1-M_{j}^{X_{j}}}{M_{j}-M_{j}^{X_{j}}}<\frac{1}{M_{j}}+\varepsilon
$$

ast $\rightarrow \infty$. 
(3) In the case of $M_{j}=1$, we take sufficiently large $t$ such that the inequality $\delta_{j}(t)<\left|c_{j}^{l}\right| \delta_{j}(t)^{\frac{1}{2}}$ holds and set $\Delta_{j}(t)=\delta_{j}(t)^{\frac{1}{2}}$. We set

$$
F_{j, t}^{l}(z)= \begin{cases}P_{j, t}^{l}(z)=c_{j}^{l}{ }^{2\left(1-\frac{\log |z|}{\log \delta_{j}(t)}\right)} z & \left(\delta_{j}(t) \leq|z| \leq \Delta_{j}(t)\right) \\ Q_{j, t}^{l}(z) & \left(\Delta_{j}(t) \leq|z| \leq 2 \Delta_{j}(t)\right), \\ h_{j}^{l}(z) & \left(2 \Delta_{j}(t) \leq|z|<1\right) .\end{cases}
$$

The functions $P_{j, t}^{l}, Q_{j, t}^{l}$ are constructed similarly as in the case of (1). On $P_{j, t}^{l}$, the above notation is obtained by changing $M_{j}, M_{j}^{X_{j}}$ to $1, \frac{1}{2}$, respectively, in (i) of the case (1). In this time,

$$
K\left(P_{j, t}^{l}\right)=\frac{\left|1-\frac{\log c_{j}^{l}}{\log \delta_{j}(t)}\right|+\left|\frac{\log c_{j}^{l}}{\log \delta_{j}(t)}\right|}{\left|1-\frac{\log c_{j}^{l}}{\log \delta_{j}(t)}\right|-\left|\frac{\log c_{j}^{l}}{\log \delta_{j}(t)}\right|}
$$

and then, $K\left(P_{j, t}^{l}\right) \rightarrow 1$ as $t \rightarrow \infty$. The function $Q_{j, t}^{l}$ also satisfies $K\left(Q_{j, t}^{l}\right) \rightarrow 1$ as $t \rightarrow \infty$. Therefore, for sufficiently large $t$, the quasiconformal mapping $F_{j, t}^{l}$ satisfies

$$
K\left(F_{j, t}^{l}\right)=\max \left\{K\left(P_{j, t}^{l}\right), K\left(Q_{j, t}^{l}\right)\right\} \rightarrow 1
$$

as $t \rightarrow \infty$.

Thus, by (1), (2) and (3), for sufficiently large $t$, we construct the quasiconformal mapping $F_{t}: Y_{t} \rightarrow Y_{t}^{\prime}$ by gluing $\left\{F_{j, t}^{l}\right\}_{j=1, \cdots, k}^{l=1,2}$. For any case of (1), (2) and (3), each $h_{j}^{l}$ is homotopic to $\left(g_{t}^{\prime} \circ f^{\prime}\right) \circ\left(g_{t} \circ f\right)^{-1}$. Each $Q_{j, t}^{l}$ satisfies $K\left(Q_{j, t}^{l}\right) \rightarrow 1$ as $t \rightarrow \infty$ and the domain $\left\{\Delta_{j}(t)<|z|<2 \Delta_{j}(t)\right\}$ has the constant modulus for any $t$. Each $P_{j, t}^{l}$ produces the twist of angle $\arg c_{j}^{l}$ in the domain $\left\{\delta_{j}(t)<|z|<\Delta_{j}(t)\right\}$ and satisfies $\left|\arg c_{j}^{1}+\arg c_{j}^{2}\right|<2 \pi$. Therefore, for sufficiently large $t$, the mapping $F_{t}$ is homotopic to $\left(g_{t}^{\prime} \circ f^{\prime}\right) \circ\left(g_{t} \circ f\right)^{-1}$. We conclude that

$$
\lim _{t \rightarrow \infty} K\left(F_{t}\right)=\lim _{t \rightarrow \infty} \max _{j=1, \cdots, k, l=1,2} K\left(F_{j, t}^{l}\right)<\max _{j=1, \cdots, k}\left\{M_{j}, \frac{1}{M_{j}}\right\}+\varepsilon .
$$

Therefore, by this lemma, for any sufficiently large $t$, the inequality

$$
\limsup _{t \rightarrow \infty} d_{T(X)}\left(r(t), r^{\prime}(t)\right) \leq \lim _{t \rightarrow \infty} \frac{1}{2} \log K\left(F_{t}\right)<\frac{1}{2} \log \left(\max _{j=1, \cdots, k}\left\{\frac{m_{j}^{\prime}}{m_{j}}, \frac{m_{j}}{m_{j}^{\prime}}\right\}+\varepsilon\right)
$$

holds. Since $\varepsilon$ is arbitrary, we are done.

3.2. Lower estimate. Next, we show that

$$
\liminf _{t \rightarrow \infty} d_{T(X)}\left(r(t), r^{\prime}(t)\right) \geq \frac{1}{2} \log \max _{j=1, \cdots, k}\left\{\frac{m_{j}^{\prime}}{m_{j}}, \frac{m_{j}}{m_{j}^{\prime}}\right\} .
$$

We use the following theorems. Let $r$ be a Teichmüller geodesic ray on $T(X)$ starting at $r(0)=[Y, f]$ and having an initial unit norm holomorphic quadratic differential $q$ on $Y$ with the corresponding measured foliation $H(q)=\sum_{j=1}^{k} b_{j} f_{*}\left(G_{j}\right) \in$ $\mathcal{M F}(Y)$ where $b_{1}, \cdots, b_{k}$ are positive real numbers and $G_{j}$ is a simple closed curve or an ergodic measure for any $j=1, \cdots, k$ such that these are projectively distinct 
and pairwise having zero intersection number. We set $m_{j}=\frac{b_{j}}{i\left(f_{*}\left(G_{j}\right), V(q)\right)}$ for any $j=1, \cdots, k$.

Theorem 3.2 ([Wal12]). If we set

$$
\mathcal{E}_{r}(\mu)=\left\{\sum_{j=1}^{k} m_{j} i\left(G_{j}, \mu\right)^{2}\right\}^{\frac{1}{2}}
$$

for any $\mu \in \mathcal{M F}(X)$, then, the following equation holds:

$$
\lim _{t \rightarrow \infty} e^{-2 t} \operatorname{Ext}_{r(t)}(\mu)=\sum_{j=1}^{k} m_{j} i\left(f_{*}\left(G_{j}\right), f_{*}(\mu)\right)^{2}=\mathcal{E}_{r}(\mu)^{2} .
$$

Furthermore, let $r^{\prime}$ be a Teichmüller geodesic ray on $T(X)$ starting at $r^{\prime}(0)=$ $\left[Y^{\prime}, f^{\prime}\right]$ and having an initial unit norm holomorphic quadratic differential $q^{\prime}$ on $Y^{\prime}$, and let $H\left(q^{\prime}\right) \in \mathcal{M F}\left(Y^{\prime}\right)$ be the corresponding measured foliation. We set $Z=\left\{\mu \in \mathcal{M F}(X)^{*} \mid \mathcal{E}_{r}(\mu)=\mathcal{E}_{r^{\prime}}(\mu)=0\right\}$.

Theorem 3.3 ([Wal12] $)$. If we can write $H\left(q^{\prime}\right)=\sum_{j=1}^{k} b_{j}^{\prime} f_{*}^{\prime}\left(G_{j}\right)$ where $b_{j}^{\prime} \geq 0$, and set $m_{j}^{\prime}=\frac{b_{j}^{\prime}}{i\left(f_{*}^{\prime}\left(G_{j}\right), V\left(q^{\prime}\right)\right)}$ for any $j=1, \cdots, k$, then the equation

$$
\sup _{\mu \in \mathcal{M F}(X)^{*-Z}} \frac{\mathcal{E}_{r^{\prime}}(\mu)^{2}}{\mathcal{E}_{r}(\mu)^{2}}=\max _{j=1, \cdots, k} \frac{m_{j}^{\prime}}{m_{j}}
$$

holds. Otherwise, the supremum is $+\infty$.

Remark. If $f_{*}^{-1}(H(q)), f_{*}^{\prime-1}\left(H\left(q^{\prime}\right)\right)$ are absolutely continuous, then $\sup \frac{\mathcal{E}_{r^{\prime}}(\mu)^{2}}{\mathcal{E}_{r}(\mu)^{2}}$ and $\sup \frac{\mathcal{E}_{r}(\mu)^{2}}{\mathcal{E}_{r^{\prime}}(\mu)^{2}}$ are both finite.

We notice that the desired inequality (1) holds for the rays $r, r^{\prime}$ which show that $f_{*}^{-1}(H(q)), f_{*}^{\prime-1}\left(H\left(q^{\prime}\right)\right)$ are absolutely continuous. So, we do not require the conditions that the rays are Jenkins-Strebel and $r(\infty)=r^{\prime}(\infty)$. The general case is used on Proposition 4.15. We write $H(q)=\sum_{j=1}^{k} b_{j} f_{*}\left(G_{j}\right), H\left(q^{\prime}\right)=\sum_{j=1}^{k} b_{j}^{\prime} f_{*}^{\prime}\left(G_{j}\right)$ where $b_{j}, b_{j}^{\prime}>0$ and set $m_{j}=\frac{b_{j}}{i\left(f_{*}\left(G_{j}\right), V(q)\right)}, m_{j}^{\prime}=\frac{b_{j}^{\prime}}{i\left(f_{*}^{\prime}\left(G_{j}\right), V\left(q^{\prime}\right)\right)}$ for any $j=$ $1, \cdots, k$. Therefore, in our case, by Kerckhoff's formula and the two theorems above,

$$
\begin{aligned}
\liminf _{t \rightarrow \infty} d_{T(X)}\left(r(t), r^{\prime}(t)\right) & =\liminf _{t \rightarrow \infty} \frac{1}{2} \log \sup _{\mu \in \mathcal{M} \mathcal{F}(X)^{*}} \frac{\operatorname{Ext}_{r^{\prime}(t)}(\mu)}{\operatorname{Ext}_{r(t)}(\mu)} \\
& \geq \frac{1}{2} \log \sup _{\mu \in \mathcal{M} \mathcal{F}(X)^{*}-Z} \liminf _{t \rightarrow \infty} \frac{e^{-2 t} \operatorname{Ext}_{r^{\prime}(t)}(\mu)}{e^{-2 t} \operatorname{Ext}_{r(t)}(\mu)} \\
& =\frac{1}{2} \log \max _{j=1, \cdots, k} \frac{m_{j}^{\prime}}{m_{j}}
\end{aligned}
$$

This inequality comes from the fact that the limit of the supremum is greater than or equal to the supremum of the limit. Because of the symmetry of the distance, the inequality (11) holds.

Remark. If $f_{*}^{-1}(H(q)), f_{*}^{\prime-1}\left(H\left(q^{\prime}\right)\right)$ are not absolutely continuous, then

$$
\liminf _{t \rightarrow \infty} d_{T(X)}\left(r(t), r^{\prime}(t)\right)=+\infty .
$$


Proof of Theorem 1.1. Because of the upper and lower estimates, we obtain the desired equation.

Corollary 1.2. For any two Jenkins-Strebel rays $r, r^{\prime}$, they are asymptotic if and only if $r, r^{\prime}$ are modularly equivalent and $r(\infty)=r^{\prime}(\infty)$.

Proof. Under the assumption of Theorem 1.1, if in addition the given rays $r, r^{\prime}$ are modularly equivalent, there is $\lambda>0$ such that $m_{j}^{\prime}=\lambda m_{j}$ for any $j=1, \cdots, k$. Then for $\alpha=-\frac{1}{2} \log \lambda$,

$$
\lim _{t \rightarrow \infty} d_{T(X)}\left(r(t), r^{\prime}(t+\alpha)\right)=\frac{1}{2} \log \max _{j=1 \cdots, k}\left\{\frac{e^{2 \alpha} m_{j}^{\prime}}{m_{j}}, \frac{m_{j}}{e^{2 \alpha} m_{j}^{\prime}}\right\}=\frac{1}{2} \log 1=0 .
$$

This means that the rays are asymptotic. Conversely, if two Jenkins-Strebel rays $r, r^{\prime}$ are asymptotic, we can set

$$
\lim _{t \rightarrow \infty} d_{T(X)}\left(r(t), r^{\prime}(t)\right)=0
$$

without loss of generality. From the above remark, $f_{*}^{-1}(H(q)), f_{*}^{\prime-1}\left(H\left(q^{\prime}\right)\right)$ are absolutely continuous. By the inequality (11), $m_{j}=m_{j}^{\prime}$ for any $j=1, \cdots, k$. Therefore, the rays are modularly equivalent. Next, we show the correspondence between the end points $r(\infty)$ and $r^{\prime}(\infty)$. The proof is similar to Proposition 2.8 ([HS07]). For any $\varepsilon>0$ and any compact neighborhood $V$ of the set of nodes in $Y_{\infty}$, we recall the neighborhood $U_{V, \varepsilon}(r(\infty))$ of $r(\infty)$ :

$U_{V, \varepsilon}(r(\infty))=\left\{[S, g] \in \hat{T}(X) \mid\right.$ there is a deformation $h: S \rightarrow Y_{\infty}$ which is $(1+\varepsilon)$-quasiconformal on $h^{-1}\left(Y_{\infty}-V\right)$ such that $g_{\infty} \circ f$ is homotopic to $\left.h \circ g\right\}$.

We set $V=V_{1} \cup \cdots \cup V_{k}$ where $V_{j}=V_{j}^{1} \cup V_{j}^{2} \cup\{p t\}, V_{j}^{l}=\left\{0<|z| \leq \iota_{j}\right\} \subset A_{j}^{l}(\infty)$, $0<\iota_{j}<1$ for any $j=1, \cdots, k$ and $l=1,2$. There exists $T>0$ such that for any $t>T$ and any $j=1, \cdots, k, \delta_{j}(t)<\iota_{j}$ and $d_{T(X)}\left(r(t), r^{\prime}(t)\right)<\frac{1}{2} \log (1+\varepsilon)$, i.e., there exists a $(1+\varepsilon)$-quasiconformal mapping $\alpha_{t}: Y_{t}^{\prime} \rightarrow Y_{t}$ such that $g_{t} \circ f$ is homotopic to $\alpha_{t} \circ g_{t}^{\prime} \circ f^{\prime}$. For any $t>T$, we want to show that $r^{\prime}(t) \in U_{V, \varepsilon}(r(\infty))$. We construct the deformation $h: Y_{t}^{\prime} \rightarrow Y_{\infty}$ as follows. For any $j=1, \cdots, k$ and $l=1,2$,

$$
\left.h\right|_{\alpha_{t}^{-1}\left(A_{j}^{l}(t)\right)}(w)= \begin{cases}\alpha_{t}(w) & \left(w \in \alpha_{t}^{-1}\left(\left\{\iota_{j} \leq|z|<1\right\}\right)\right), \\ h_{j, t}\left(\left|\alpha_{t}(w)\right|\right) e^{i \arg \alpha_{t}(w)} & \left(w \in \alpha_{t}^{-1}\left(\left\{\delta_{j}(t)<|z|<\iota_{j}\right\}\right)\right), \\ p t & \left(w \in \alpha_{t}^{-1}\left(\left\{|z|=\delta_{j}(t)\right\}\right)\right),\end{cases}
$$

where $h_{j, t}:\left(\delta_{j}(t), \iota_{j}\right) \rightarrow\left(0, \iota_{j}\right)$ is an arbitrary monotonously increasing diffeomorphism. The mapping $\left.h\right|_{\alpha_{t}^{-1}\left(A_{j}^{l}(t)\right)}$ is equal to the $(1+\varepsilon)$-quasiconformal mapping $\alpha_{t}$ on $\left.h\right|_{\alpha_{t}^{-1}\left(A_{j}^{l}(t)\right)}{ }^{-1}\left(A_{j}^{l}(\infty)-V_{j}^{l}\right)$. Since $h$ is homotopic to $g_{\infty} \circ g_{t}^{-1} \circ \alpha_{t}$, we obtain that $h \circ g_{t}^{\prime} \circ f^{\prime}$ is homotopic to $g_{\infty} \circ f$. This means that $r^{\prime}(t) \in U_{V, \varepsilon}(r(\infty))$ for any $t>T$. Since $\varepsilon$ and $V$ are arbitrary, we conclude that $r(\infty)=r^{\prime}(\infty)$.

\section{The Detour Metric}

In this section, we obtain the minimum value of the equation of Theorem 1.1 when we shift the initial points of the given two rays. This value is represented by the detour metric between the end points of the rays in the Gardiner-Masur boundary of $T(X)$. 
4.1. The horofunction boundary of metric spaces and the detour metric. We recall the definition of the detour metric in the case of a general metric space, and refer the reader to Wal11] for more details. First, we consider the horofunction compactification of a metric space. This was given by Gromov Gro81. Let $(X, d)$ be a metric space, and $b$ a base point of $X$. The distance $d$ on $X$ is called proper if any closed ball with respect to $d$ is compact and geodesic if for any two points in $X$, there exists a geodesic which joins them. Suppose that the distance $d$ is proper and geodesic. It is well known that any Teichmüller distance has these properties. We denote by $C(X)$ the set of all continuous functions of $X$ into $\mathbb{R}$ which is equipped with the topology of the uniform convergence on any compact set of $X$. We define a mapping $\psi: X \rightarrow C(X)$ by $z \mapsto\left\{\psi_{z}(x):=d(x, z)-d(b, z)\right\}_{x \in X}$.

Theorem 4.1 (Gro81]). The mapping $\psi$ is an embedding and the set $\psi(X)$ is relatively compact on $C(X)$.

By this theorem, the space $X$ is identified with $\psi(X)$. The closure $\overline{\psi(X)}$ is called the horofunction compactification of $X$. The boundary $X(\infty)=\overline{\psi(X)}-\psi(X)$ is called the horofunction boundary of $X$. We call $\xi \in X(\infty)$ a horofunction. We can denote by $X \cup X(\infty)$ the horofunction compactification of $X$.

Remark. The topological space $X \cup X(\infty)$ satisfies the first countability axiom.

Definition 4.2. For any $\xi, \xi^{\prime} \in X(\infty)$, we define the detour cost

$$
H\left(\xi, \xi^{\prime}\right)=\sup _{W \ni \xi} \inf _{x \in W \cap X}\left(d(b, x)+\xi^{\prime}(x)\right),
$$

where $W$ ranges over all neighborhoods of $\xi$ in $X \cup X(\infty)$.

There is another definition of the detour cost.

Definition 4.3. For any $\xi, \xi^{\prime} \in X(\infty)$,

$$
H\left(\xi, \xi^{\prime}\right)=\inf _{\gamma} \liminf _{t \rightarrow \infty}\left(d(b, \gamma(t))+\xi^{\prime}(\gamma(t))\right),
$$

where $\gamma$ ranges over all paths $\gamma: \mathbb{R}_{\geq 0} \rightarrow X$ which converge to $\xi$.

Definition 4.4. Let $A \subset \mathbb{R}_{\geq 0}$ be an unbounded set which contains 0. A mapping $r: A \rightarrow X$ is called an almost geodesic on $X$ if for any $\varepsilon>0$, there exists $T \geq 0$ such that for any $s, t \in A$ with $T \leq s \leq t$,

$$
|d(r(0), r(s))+d(r(s), r(t))-t|<\varepsilon .
$$

Any geodesic is an almost geodesic. Rieffel proved that any almost geodesic on $X$ converges to a point in $X(\infty)$ ([Rie02]). Let $X_{B}(\infty) \subset X(\infty)$ be the set of end points of all almost geodesics. Any $\xi \in X_{B}(\infty)$ is called a Busemann point.

Proposition 4.5 (Wal1] $)$. For any $\xi, \xi^{\prime}, \xi^{\prime \prime} \in X(\infty)$ and $x \in X$, the detour cost $H$ satisfies the following properties:

(1) $\xi^{\prime}(x) \leq H\left(\xi, \xi^{\prime}\right)+\xi(x)$,

(2) $H(\xi, \xi)=0$ if and only if $\xi \in X_{B}(\infty)$,

(3) $H\left(\xi, \xi^{\prime}\right) \geq 0$,

(4) $H\left(\xi, \xi^{\prime \prime}\right) \leq H\left(\xi, \xi^{\prime}\right)+H\left(\xi^{\prime}, \xi^{\prime \prime}\right)$.

By this proposition, for any $\xi, \xi^{\prime} \in X_{B}(\infty)$, the symmetrization $H\left(\xi, \xi^{\prime}\right)+$ $H\left(\xi^{\prime}, \xi\right)$ satisfies the axiom of the distance.

Definition 4.6. For any $\xi, \xi^{\prime} \in X_{B}(\infty)$, 


$$
\delta\left(\xi, \xi^{\prime}\right)=H\left(\xi, \xi^{\prime}\right)+H\left(\xi^{\prime}, \xi\right)
$$

is a (possibly $+\infty$-valued) distance on $X_{B}(\infty)$. This $\delta$ is called the detour metric for $(X, d)$ and the base point $b \in X$.

4.2. The Gardiner-Masur boundary of Teichmüller spaces. The GardinerMasur compactification and the Gardiner-Masur boundary of a Teichmüller space were induced by Gardiner and Masur GM91. Liu and Su LS12 showed that the horofunction compactification of the Teichmüller space with the Teichmüller distance is the same as the Gardiner-Masur compactification. Let $T(X)$ be a Teichmüller space of $X$. We define a mapping $\tilde{\phi}: T(X) \rightarrow \mathbb{R}_{\geq 0}^{\mathcal{S}}$ by $p \mapsto\left\{\operatorname{Ext}_{p}^{\frac{1}{2}}(\gamma)\right\}_{\gamma \in \mathcal{S}}$, and denote by $\pi: \mathbb{R}_{\geq 0}^{\mathcal{S}}-\{0\} \rightarrow \mathrm{PR}_{\geq 0}^{\mathcal{S}}$ a natural projection.

Theorem 4.7 ([GM91]). The composition $\phi=\pi \circ \tilde{\phi}: T(X) \rightarrow \mathrm{P} \mathbb{R}_{\geq 0}^{\mathcal{S}}$ is an embedding and the closure $\overline{\phi(T(X))}$ is a compact set.

This closure is called the Gardiner-Masur compactification of $T(X)$ and we denote by $\overline{T(X)}^{G M}=\overline{\phi(T(X))}$. The boundary $\partial_{G M} T(X)=\overline{\phi(T(X))}-\phi(T(X))$ is called the Gardiner-Masur boundary of $T(X)$.

We set the base point $b=[X, i d] \in T(X)$. For any $p \in T(X)$ and any $\mu \in$ $\mathcal{M F}(X)$, we define

$$
\mathcal{E}_{p}(\mu)=\left\{\frac{\operatorname{Ext}_{p}(\mu)}{K_{p}}\right\}^{\frac{1}{2}},
$$

where $K_{p}=e^{2 d_{T(X)}(b, p)}$. By the definition of the Gardiner-Masur compactification, the function $\pi\left(\left\{\mathcal{E}_{p}(\gamma)\right\}_{\gamma \in \mathcal{S}}\right)$ corresponds to $p \in T(X)$.

Proposition 4.8 (Miy08]). For any $\xi \in \partial_{G M} T(X)$, there exists a continuous function $\mathcal{E}_{\xi}: \mathcal{M F}(X) \rightarrow \mathbb{R}_{\geq 0}$ which satisfies the following properties:

(1) $\mathcal{E}_{\xi}(t \mu)=t \mathcal{E}_{\xi}(\mu)$ for any $t \geq 0$ and any $\mu \in \mathcal{M F}(X)$,

(2) $\pi\left(\left\{\mathcal{E}_{p}(\gamma)\right\}_{\gamma \in \mathcal{S}}\right)$ corresponds to $\xi \in \partial_{G M} T(X)$,

(3) If a sequence $\left\{x_{n}\right\} \subset T(X)$ converges to $\xi \in \partial_{G M} T(X)$, then there exists a subsequence $\left\{x_{n_{j}}\right\} \subset\left\{x_{n}\right\}$ and $t_{0}>0$ which does not depend on $\mathcal{M F}(X)$ such that $\mathcal{E}_{x_{n_{j}}}$ converges to $t_{0} \mathcal{E}_{\xi}$ uniformly on any compact set of $\mathcal{M F}(X)$.

For any $p \in \overline{T(X)}^{G M}$, we define

$$
Q(p)=\sup _{\nu \in \mathcal{M} \mathcal{F}(X)^{*}} \frac{\mathcal{E}_{p}(\nu)}{\operatorname{Ext}_{b}^{\frac{1}{2}}(\nu)}
$$

and for any $\mu \in \mathcal{M F}(X)$,

$$
\mathcal{L}_{p}(\mu)=\frac{\mathcal{E}_{p}(\mu)}{Q(p)}
$$

Proposition 4.9 ([LS12 $)$. For any $\left\{p_{n}\right\} \subset \overline{T(X)}^{G M}$ and any $p \in \overline{T(X)}^{G M}, p_{n}$ converges to $p$ as $n \rightarrow \infty$ if and only if $\mathcal{L}_{p_{n}}$ converges to $\mathcal{L}_{p}$ uniformly on any compact set of $\mathcal{M F}(X)$ as $n \rightarrow \infty$. 
For any $p \in \overline{T(X)}^{G M}$, we define a function $\psi_{p}: T(X) \rightarrow \mathbb{R}$ by

for any $x \in T(X)$.

$$
\psi_{p}(x)=\log \sup _{\mu \in \mathcal{M} \mathcal{F}(X) *} \frac{\mathcal{L}_{p}(\mu)}{\operatorname{Ext}_{x}^{\frac{1}{2}}(\mu)}
$$

Remark. By the definition and Kerckhoff's formula, if $p \in T(X)$, then

$$
\begin{aligned}
\psi_{p}(x) & =\log \sup _{\mu \in \mathcal{M} \mathcal{F}(X)^{*}} \frac{\mathcal{E}_{p}(\mu)}{\operatorname{Ext}_{x}^{\frac{1}{2}}(\mu)}-\log \sup _{\mu \in \mathcal{M} \mathcal{F}(X)^{*}} \frac{\mathcal{E}_{p}(\mu)}{\operatorname{Ext}_{b}^{\frac{1}{2}}(\mu)} \\
& =\log \sup _{\mu \in \mathcal{M} \mathcal{F}(X) *} \frac{\operatorname{Ext}_{p}^{\frac{1}{2}}(\mu)}{\operatorname{Ext}_{x}^{\frac{1}{2}}(\mu)}-\log \sup _{\mu \in \mathcal{M} \mathcal{F}(X) *} \frac{\operatorname{Ext}_{p}^{\frac{1}{2}}(\mu)}{\operatorname{Ext}_{b}^{\frac{1}{2}}(\mu)} \\
& =d_{T(X)}(x, p)-d_{T(X)}(b, p)
\end{aligned}
$$

for any $x \in X$. Thus, we can consider the horofunction compactification of $T(X)$ by the function $\psi_{p}$ for any $p \in T(X)$.

The following is deduced from Proposition 4.9.

Theorem 4.10 ([LS12] $)$. We define a mapping $\psi: \overline{T(X)}^{G M} \rightarrow C(T(X))$ by $p \mapsto$ $\psi_{p}$. Then $\psi$ is injective and continuous. In particular, $\overline{T(X)}^{G M}$ and $\psi\left(\overline{T(X)}^{G M}\right)$ are homeomorphic. Furthermore, $\psi\left(\overline{T(X)}^{G M}\right)=\overline{\psi(T(X))}$.

Therefore, the horofunction compactification $\overline{\psi(T(X))}$ of $T(X)$ can be identified with the Gardiner-Masur compactification $\overline{T(X)}^{G M}$. We denote by $T(X) \cup$ $T(X)(\infty)$ the horofunction compactification of $T(X)$. Then, we can assume that $T(X)(\infty)=\partial_{G M} T(X)$.

4.3. The detour metric for the Teichmüller distance. We consider the detour metric in the case of the Teichmüller space with the Teichmüller distance. Its representation is given by Walsh Wal12. We notice that there exist non-Busemann points on $\partial_{G M} T(X)$ if $3 g-3+n>1$. This result is proved by Miyachi Miy11.

Theorem 4.11 ([Wal12]). For any point $p$ in $T(X)$ and any Busemann point $\xi$, there exists a unique Teichmüller geodesic ray on $T(X)$ starting at $p$ and converging to $\xi$.

By this theorem, we can assume that the set of all Busemann points consists of end points of all Teichmüller geodesic rays.

Theorem 4.12 (Wal12). Let $r, r^{\prime}$ be Teichmüller geodesic rays on $T(X)$ converging to Busemann points $\xi, \xi^{\prime}$, respectively. Then, the rays $r, r^{\prime}$ are modularly equivalent if and only if $\xi=\xi^{\prime}$.

Let $\xi, \xi^{\prime}$ be Busemann points and $r, r^{\prime}$ Teichmüller geodesic rays on $T(X)$ starting at $b=[X, i d]$ and converging to $\xi, \xi^{\prime}$, respectively. We denote by $q, q^{\prime}$ the unit norm holomorphic quadratic differentials on $X$ corresponding to the given rays $r, r^{\prime}$, respectively. If the measured foliations $H(q), H\left(q^{\prime}\right) \in \mathcal{M F}(X)$ are absolutely continuous, then we can write $H(q)=\sum_{j=1}^{k} b_{j} G_{j}, H\left(q^{\prime}\right)=\sum_{j=1}^{k} b_{j}^{\prime} G_{j}$ where $b_{j}, b_{j}^{\prime}>0$ and $G_{j}$ is a simple closed curve or an ergodic measure for any $j=1, \cdots, k$ such that these are projectively distinct and pairwise having zero intersection number. We set $m_{j}=\frac{b_{j}}{i\left(G_{j}, V(q)\right)}, m_{j}^{\prime}=\frac{b_{j}^{\prime}}{i\left(G_{j}, V\left(q^{\prime}\right)\right)}$ for any $j=1, \cdots, k$. 
Theorem 4.13 (Wal12]). If $H(q), H\left(q^{\prime}\right)$ are absolutely continuous, then the detour metric $\delta$ between $\xi$ and $\xi^{\prime}$ is represented by

$$
\delta\left(\xi, \xi^{\prime}\right)=\frac{1}{2} \log \max _{j=1, \cdots, k} \frac{m_{j}^{\prime}}{m_{j}}+\frac{1}{2} \log \max _{j=1, \cdots, k} \frac{m_{j}}{m_{j}^{\prime}} .
$$

If $H(q), H\left(q^{\prime}\right)$ are not absolutely continuous, then $\delta\left(\xi, \xi^{\prime}\right)=+\infty$.

In Theorem 4.13, for any given Busemann points, Walsh showed the representation of the detour metric between them. Now, for any given rays, we obtain the representation of the detour metric between the end points of the rays. Let $r, r^{\prime}$ be Teichmüller geodesic rays on $T(X)$ starting at $r(0)=[Y, f], r^{\prime}(0)=\left[Y^{\prime}, f^{\prime}\right]$ and having initial unit norm holomorphic quadratic differentials $q, q^{\prime}$ on $Y, Y^{\prime}$ and converging to Busemann points $\xi, \xi^{\prime}$, respectively. If the measured foliations $f_{*}^{-1}(H(q)), f_{*}^{\prime-1}\left(H\left(q^{\prime}\right)\right) \in \mathcal{M F}(X)$ are absolutely continuous, then we can write $f_{*}^{-1}(H(q))=\sum_{j=1}^{k} b_{j} G_{j}, f_{*}^{\prime-1}(H(q))=\sum_{j=1}^{k} b_{j}^{\prime} G_{j}$ where $b_{j}, b_{j}^{\prime}>0, G_{j}$ is a simple closed curve or an ergodic measure for any $j=1, \cdots, k$ such that these are projectively distinct and pairwise having zero intersection number. We set $m_{j}=\frac{b_{j}}{i\left(f_{*}\left(G_{j}\right), V(q)\right)}, m_{j}^{\prime}=\frac{b_{j}^{\prime}}{i\left(f_{*}^{\prime}\left(G_{j}\right), V\left(q^{\prime}\right)\right)}$ for any $j=1, \cdots, k$. We combine Theorems $4.11,4.12$ and 4.13 to obtain the following.

Proposition 4.14. If $f_{*}^{-1}(H(q)), f_{*}^{\prime-1}\left(H\left(q^{\prime}\right)\right)$ are absolutely continuous, then the detour metric between $\xi$ and $\xi^{\prime}$ is also represented by

$$
\delta\left(\xi, \xi^{\prime}\right)=\frac{1}{2} \log \max _{j=1, \cdots, k} \frac{m_{j}^{\prime}}{m_{j}}+\frac{1}{2} \log \max _{j=1, \cdots, k} \frac{m_{j}}{m_{j}^{\prime}} .
$$

If $f_{*}^{-1}(H(q)), f_{*}^{\prime-1}\left(H\left(q^{\prime}\right)\right)$ are not absolutely continuous, then $\delta\left(\xi, \xi^{\prime}\right)=+\infty$.

Proof. By Theorem 4.11, there exist two Teichmüller geodesic rays $s, s^{\prime}$ starting at $b=[X, i d]$ and having initial unit norm holomorphic quadratic differentials $\varphi, \varphi^{\prime}$ on $X$ and converging to Busemann points $\xi, \xi^{\prime}$, respectively. By Theorem 4.12 , the two pairs $r, s$ and $r^{\prime}, s^{\prime}$ are both modularly equivalent. If the measured foliations $f_{*}^{-1}(H(q)), f_{*}^{\prime-1}\left(H\left(q^{\prime}\right)\right)$ are absolutely continuous, then the measured foliations $H(\varphi), H\left(\varphi^{\prime}\right) \in \mathcal{M F}(X)$ are also absolutely continuous, and can be written as $H(\varphi)=\sum_{j=1}^{k} c_{j} G_{j}, H\left(\varphi^{\prime}\right)=\sum_{j=1}^{k} c_{j}^{\prime} G_{j}$ where $c_{j}, c_{j}^{\prime}>0$ for any $j=1, \cdots, k$. Let $n_{j}=\frac{c_{j}}{i\left(G_{j}, V(\varphi)\right)}, n_{j}^{\prime}=\frac{c_{j}^{\prime}}{i\left(G_{j}, V\left(\varphi^{\prime}\right)\right)}$ for any $j=1, \cdots, k$, then there are $\lambda, \lambda^{\prime}>0$ such that $n_{j}=\lambda m_{j}, n_{j}^{\prime}=\lambda^{\prime} m_{j}^{\prime}$, respectively. Therefore, by Theorem 4.13 ,

$$
\begin{aligned}
\delta\left(\xi, \xi^{\prime}\right) & =\frac{1}{2} \log \max _{j=1, \cdots, k} \frac{n_{j}^{\prime}}{n_{j}}+\frac{1}{2} \log \max _{j=1, \cdots, k} \frac{n_{j}}{n_{j}^{\prime}} \\
& =\frac{1}{2} \log \max _{j=1, \cdots, k} \frac{m_{j}^{\prime}}{m_{j}}+\frac{1}{2} \log \max _{j=1, \cdots, k} \frac{m_{j}}{m_{j}^{\prime}} .
\end{aligned}
$$

If the measured foliations $f_{*}^{-1}(H(q)), f_{*}^{\prime-1}\left(H\left(q^{\prime}\right)\right)$ are not absolutely continuous, then $H(\varphi), H\left(\varphi^{\prime}\right)$ are not also absolutely continuous, and we conclude that $\delta\left(\xi, \xi^{\prime}\right)=$ $+\infty$.

We suppose that two rays $r, r^{\prime}$ show that $f_{*}^{-1}(H(q)), f_{*}^{\prime-1}\left(H\left(q^{\prime}\right)\right)$ are absolutely continuous. Let $\xi, \xi^{\prime}$ be Busemann points corresponding to the end points of $r, r^{\prime}$ 
respectively. By the lower estimate of Theorem 1.1, and Proposition 4.14,

$$
\text { (2) } \begin{aligned}
\liminf _{t \rightarrow \infty} d_{T(X)}\left(r(t), r^{\prime}(t)\right) & \geq \frac{1}{2} \log \max _{j=1, \cdots, k}\left\{\frac{m_{j}^{\prime}}{m_{j}}, \frac{m_{j}}{m_{j}^{\prime}}\right\} \\
& \geq \frac{1}{2} \log \left(\max _{j=1, \cdots, k} \sqrt{\frac{m_{j}^{\prime}}{m_{j}}} \cdot \max _{j=1, \cdots, k} \sqrt{\frac{m_{j}}{m_{j}^{\prime}}}\right) \\
& =\frac{1}{2}\left(\frac{1}{2} \log \max _{j=1, \cdots, k} \frac{m_{j}^{\prime}}{m_{j}}+\frac{1}{2} \log \max _{j=1, \cdots, k} \frac{m_{j}}{m_{j}^{\prime}}\right) \\
& =\frac{1}{2} \delta\left(\xi, \xi^{\prime}\right) .
\end{aligned}
$$

Now, we consider the minimum value of the equation of Theorem 1.1 when we shift the initial points of the given rays $r, r^{\prime}$.

Proposition 1.3. Under the assumption of Theorem 1.1, the minimum of the limit value of the distance between the given rays $r(t), r^{\prime}(t)$ when we shift the initial points $r(0), r^{\prime}(0)$ is given by $\frac{1}{2} \delta\left(\xi, \xi^{\prime}\right)$ where $\xi, \xi^{\prime}$ are the end points of the rays $r$, $r^{\prime}$ in the Gardiner-Masur boundary of $T(X)$, respectively.

Proof. By Theorem 1.1 and the inequality (2), we see that

$$
\begin{aligned}
\lim _{t \rightarrow \infty} d_{T(X)}\left(r(t), r^{\prime}(t)\right) & =\frac{1}{2} \log \max _{j=1, \cdots, k}\left\{\frac{m_{j}^{\prime}}{m_{j}}, \frac{m_{j}}{m_{j}^{\prime}}\right\} \\
& \geq \frac{1}{2} \delta\left(\xi, \xi^{\prime}\right) .
\end{aligned}
$$

We notice that the detour metric is invariant when we shift the initial points of the rays $r, r^{\prime}$. The equality holds if we set

$$
\beta=\frac{1}{4} \log \frac{\max _{j=1, \cdots, k} \frac{m_{j}}{m_{j}^{\prime}}}{\max _{j=1, \cdots, k} \frac{m_{j}^{\prime}}{m_{j}}}
$$

and consider the rays $r(t), r^{\prime}(t+\beta)$. In this situation, we compute that

$$
\max _{j=1, \cdots, k} \frac{e^{2 \beta} m_{j}^{\prime}}{m_{j}}=\max _{j=1, \cdots, k}\left\{\frac{\sqrt{\max _{j=1, \cdots, k} \frac{m_{j}}{m_{j}^{\prime}}} \cdot m_{j}^{\prime}}{\sqrt{\max _{j=1, \cdots, k} \frac{m_{j}^{\prime}}{m_{j}}} \cdot m_{j}}\right\}=\sqrt{\max _{j=1, \cdots, k} \frac{m_{j}^{\prime}}{m_{j}}} \cdot \sqrt{\max _{j=1, \cdots, k} \frac{m_{j}}{m_{j}^{\prime}}}
$$

and, similarly,

$$
\max _{j=1, \cdots, k} \frac{m_{j}}{e^{2 \beta} m_{j}^{\prime}}=\sqrt{\max _{j=1, \cdots, k} \frac{m_{j}^{\prime}}{m_{j}}} \cdot \sqrt{\max _{j=1, \cdots, k} \frac{m_{j}}{m_{j}^{\prime}}} .
$$


Therefore, we conclude that

$$
\begin{aligned}
\lim _{t \rightarrow \infty} d_{T(X)}\left(r(t), r^{\prime}(t+\beta)\right) & =\frac{1}{2} \log \max _{j=1, \cdots, k}\left\{\frac{e^{2 \beta} m_{j}^{\prime}}{m_{j}}, \frac{m_{j}}{e^{2 \beta} m_{j}^{\prime}}\right\} \\
& =\frac{1}{2}\left(\frac{1}{2} \log \max _{j=1, \cdots, k} \frac{m_{j}^{\prime}}{m_{j}}+\frac{1}{2} \log \max _{j=1, \cdots, k} \frac{m_{j}}{m_{j}^{\prime}}\right) \\
& =\frac{1}{2} \delta\left(\xi, \xi^{\prime}\right) .
\end{aligned}
$$

We can also obtain the following. In Proposition 4.15, we do not require the conditions that the given rays are Jenkins-Strebel.

Proposition 4.15. If two rays $r, r^{\prime}$ are asymptotic, then the end points show that $\xi=\xi^{\prime}$ and the rays are modularly equivalent.

Proof. From the remark in the previous section, the measured foliations $f_{*}^{-1}(H(q))$, $f_{*}^{\prime-1}\left(H\left(q^{\prime}\right)\right)$ are absolutely continuous. Then, the conclusion follows from Theorem 4.12 and the inequality (2).

\section{The tables of the ClASSificAtion FOR THE BehaVior OF TWO TEICHMÜLLER RAYS}

In this section, we give the tables of the classification of the conditions under which given two Teichmüller geodesic rays are bounded, diverge, or are asymptotic.

Let $r, r^{\prime}$ be two Teichmüller geodesic rays on $T(X)$ starting at $r(0)=[Y, f]$, $r^{\prime}(0)=\left[Y^{\prime}, f^{\prime}\right]$ and having initial unit norm holomorphic quadratic differentials $q, q^{\prime}$ on $Y, Y^{\prime}$, and let $H(q), H\left(q^{\prime}\right)$ be measured foliations corresponding to $q, q^{\prime}$, respectively. We set $H=f_{*}^{-1}(H(q)), H^{\prime}=f_{*}^{\prime-1}\left(H\left(q^{\prime}\right)\right)$. In the following tables, the notation "top.equi." means topologically equivalent, "abs.conti." means absolutely continuous, "J-S." means Jenkins-Strebel, "u.e,." means uniquely ergodic, and "mod.equi." means modularly equivalent:

$$
H, H^{\prime}:\left\{\begin{array}{l}
\text { top.equi. and }\left\{\begin{array}{l}
\text { abs.conti. } \Rightarrow \text { bounded Iva01] } \\
\text { not abs.conti. } \Rightarrow \text { diverge [LM10] }
\end{array}\right. \\
\text { not top.equi. and } i\left(H, H^{\prime}\right)\left\{\begin{array}{l}
\neq 0 \Rightarrow \text { diverge [Iva01] } \\
=0 \Rightarrow \text { diverge [LM10] }
\end{array}\right.
\end{array}\right.
$$

By the above table,

$$
r, r^{\prime} \text { : bounded } \Longleftrightarrow H, H^{\prime} \text { : abs.conti. }
$$

If $H, H^{\prime}$ are absolutely continuous and Jenkins-Strebel, we denote by $r(\infty), r^{\prime}(\infty)$ the end points of the rays $r, r^{\prime}$ in the augmented Teichmüller space $\hat{T}(X)$, respectively. 
$H, H^{\prime}$ : abs.conti. and $\left\{\begin{array}{l}\text { J-S. and }\left\{\begin{array}{l}r, r^{\prime} \text { : mod.equi. and } r(\infty)=r^{\prime}(\infty) \\ \Rightarrow \text { asymptotic }(\mathrm{Cor} 1.2) \\ \text { otherwise } \\ \Rightarrow \text { bounded but not asymptotic (Cor 1.2) }\end{array}\right. \\ \text { not J-S. and }\left\{\begin{array}{l}\text { u.e., and } \Gamma_{q}, \Gamma_{q^{\prime}} \text { have no simple } \\ \text { closed curves } \Rightarrow \text { asymptotic [Mas80 } \\ \text { otherwise } \Rightarrow \text { unknown }\end{array}\right.\end{array}\right.$

Let $\xi, \xi^{\prime}$ be the end points of the rays $r, r^{\prime}$ in the Gardiner-Masur boundary $\partial_{G M} T(X)$, respectively.

$$
r, r^{\prime} \text { : asymptotic } \underset{\text { (Prop 4.15] }}{\Longrightarrow} \xi=\xi^{\prime} \underset{\text { Wal12 }}{\Longleftrightarrow} \quad r, r^{\prime}: \text { mod.equi. }
$$

\section{ACKNOWLEDGMENTS}

The author is deeply grateful to Professor Hiroshige Shiga and Professor Hideki Miyachi for giving many insightful comments and warm encouragement. He also thanks Professor Hiroki Sato and Professor Yoshihide Okumura, who led him to the field of complex analysis. The author also expresses gratitude to the referees for their careful reading of this paper and for giving useful comments. This work was supported by the Global COE Program "Computationalism as Foundation for the Sciences".

\section{REFERENCES}

[Abi77] William Abikoff, Degenerating families of Riemann surfaces, Ann. of Math. (2) 105 (1977), no. 1, 29-44. MR0442293 (56 \#679)

[FLP79] Albert Fathi, François Laudenbach, and Valentin Poénaru. Travaux de Thurston sur les surfaces, volume 66 of Astérisque. Société Mathématique de France, Paris, 1979. Séminaire Orsay, With an English summary.

[FM10] Benson Farb and Howard Masur, Teichmüller geometry of moduli space, I: distance minimizing rays and the Deligne-Mumford compactification, J. Differential Geom. 85 (2010), no. 2, 187-227. MR2732976 (2011k:30053)

[GM91] Frederick P. Gardiner and Howard Masur, Extremal length geometry of Teichmüller space, Complex Variables Theory Appl. 16 (1991), no. 2-3, 209-237. MR.1099913 (92f:32034)

[Gro81] M. Gromov, Hyperbolic manifolds, groups and actions, Brook Conference (State Univ. New York, Stony Brook, N.Y., 1978), Ann. of Math. Stud., vol. 97, Princeton Univ. Press, Princeton, NJ, 1981, pp. 183-213. MR624814 (82m:53035)

[Gup11] Subhojoy Gupta. Asymptoticity of grafting and Teichmüller rays I. arXiv:1109.5365v1, 2011.

[HM79] John Hubbard and Howard Masur, Quadratic differentials and foliations, Acta Math. 142 (1979), no. 3-4, 221-274, DOI 10.1007/BF02395062. MR523212 (80h:30047)

[HS07] Frank Herrlich and Gabriela Schmithüsen, On the boundary of Teichmüller disks in Teichmüller and in Schottky space, Handbook of Teichmüller theory. Vol. I, IRMA Lect. Math. Theor. Phys., vol. 11, Eur. Math. Soc., Zürich, 2007, pp. 293-349, DOI 10.4171/029-1/7. MR2349673(2009b:30092)

[IT92] Y. Imayoshi and M. Taniguchi, An introduction to Teichmüller spaces, Springer-Verlag, Tokyo, 1992. Translated and revised from the Japanese by the authors. MR.1215481 (94b:32031) 
[Iva92] Nikolai V. Ivanov, Subgroups of Teichmüller modular groups, Translations of Mathematical Monographs, vol. 115, American Mathematical Society, Providence, RI, 1992. Translated from the Russian by E. J. F. Primrose and revised by the author. MR 1195787 (93k:57031)

[Iva01] Nikolai V. Ivanov, Isometries of Teichmüller spaces from the point of view of Mostow rigidity, Topology, ergodic theory, real algebraic geometry, Amer. Math. Soc. Transl. Ser. 2, vol. 202, Amer. Math. Soc., Providence, RI, 2001, pp. 131-149. MR1819186 (2001m:30057)

[Ker80] Steven P. Kerckhoff, The asymptotic geometry of Teichmüller space, Topology 19 (1980), no. 1, 23-41, DOI 10.1016/0040-9383(80)90029-4. MR559474 (81f:32029)

[KH95] Anatole Katok and Boris Hasselblatt, Introduction to the modern theory of dynamical systems, Encyclopedia of Mathematics and its Applications, vol. 54, Cambridge University Press, Cambridge, 1995. With a supplementary chapter by Katok and Leonardo Mendoza. MR1326374 (96c:58055)

[LM10] Anna Lenzhen and Howard Masur, Criteria for the divergence of pairs of Teichmüller geodesics, Geom. Dedicata 144 (2010), 191-210, DOI 10.1007/s10711-0099397-7. MR2580426 (2011a:32022)

[LS12] Lixin Liu and Weixu Su. The horofunction compactification of Teichmüller metric. arXiv:1012.0409v4, 2012.

[Mas75] Howard Masur, On a class of geodesics in Teichmüller space, Ann. of Math. (2) 102 (1975), no. 2, 205-221. MR0385173 (52 \#6038)

[Mas80] Howard Masur, Uniquely ergodic quadratic differentials, Comment. Math. Helv. 55 (1980), no. 2, 255-266, DOI 10.1007/BF02566685. MR576605 (82a:32031)

[Miy08] Hideki Miyachi, Teichmüller rays and the Gardiner-Masur boundary of Teichmüller space, Geom. Dedicata 137 (2008), 113-141, DOI 10.1007/s10711-008-9289-2. MR:2449148 (2009m:30093)

[Miy11] Hideki Miyachi. Teichmüller space has non-Busemann points. arXiv:1105.3070v1, 2011.

[Ree81] Mary Rees, An alternative approach to the ergodic theory of measured foliations on surfaces, Ergodic Theory Dynamical Systems 1 (1981), no. 4, 461-488 (1982). MR662738 (84c:58065)

[Rie02] Marc A. Rieffel, Group $C^{*}$-algebras as compact quantum metric spaces, Doc. Math. 7 (2002), 605-651 (electronic). MR 2015055 (2004k:22009)

[Str84] Kurt Strebel, Quadratic differentials, Ergebnisse der Mathematik und ihrer Grenzgebiete (3) [Results in Mathematics and Related Areas (3)], vol. 5, Springer-Verlag, Berlin, 1984. MR743423 (86a:30072)

[Wal11] Cormac Walsh, The action of a nilpotent group on its horofunction boundary has finite orbits, Groups Geom. Dyn. 5 (2011), no. 1, 189-206, DOI 10.4171/GGD/122. MR2763785 (2011m:20101)

[Wal12] Cormac Walsh, The asymptotic geometry of the Teichmüller metric, arXiv:1210.5565v1, 2012 .

Department of Mathematics, Tokyo Institute of Technology, 2-12-1 Ookayama, Meguroku, TOKYO 152-8551, JAPAN

E-mail address: amano.m.ab@m.titech.ac.jp 